\title{
Unentangled Vitrimer Melts: Interplay between Chain Relaxation and Cross-link Exchange Controls Linear Rheology
}

\author{
Ralm Ricarte ${ }^{*, \dagger}$ and Sachin Shanbhag ${ }^{*, \dagger}$ \\ $\dagger$ Department of Chemical and Biomedical Engineering, FAMU-FSU College of Engineering, \\ Tallahassee, FL 32310. \\ $\ddagger$ Department of Scientific Computing, Florida State University, Tallahassee, FL 32306 \\ E-mail: rricarte@eng.famu.fsu.edu; sshanbhag@fsu.edu \\ Phone: +1 (850) 410-6170; +1 (850) 644-6548
}

\begin{abstract}
Vitrimers are polymer networks that engage in dynamic associative exchange reactions. Their covalent cross-links preserve network connectivity but permit topology fluctuations, making them both insoluble and processable. Here, we use a sticky Rouse model approach to elucidate structure-viscoelasticity relationships for unentangled vitrimer melts. Two different versions of the sticky Rouse model are explored: the simplified sticky Rouse (SSR) and the inhomogeneous Rouse (IHR). Unlike the SSR, the IHR model accounts for interactions between slow modes that arise due to cross-linking and fast Rouse modes of the underlying polymer chain. First, we identify the conditions where the SSR sufficiently approximates the IHR. Then, we use the IHR to explore the influence of structure and temperature on the zeroshear viscosity $\left(\eta_{0}\right)$ and characteristic relaxation time $\left(\tau^{*}\right)$. Vitrimers with uniform and random cross-link distributions exhibit larger $\eta_{0}$ and $\tau^{*}$ than gradient and blocky types. Polydimethylsiloxane vitrimer (which has a flexible backbone) shows an Arrhenius temperature dependence
\end{abstract}


for $\eta_{0}$, while polystyrene vitrimer (which has a rigid backbone) is only Arrhenius at high temperatures. For stress relaxation measurements, the short time dynamics represent monomer friction, while the long time dynamics encompass a combination of network strand relaxation and cross-link exchange. Due to the different temperature dependences of the processes, timetemperature superposition fails. The effective rheological activation energy can be estimated a priori from the cross-link exchange activation energy and backbone Williams-Landel-Ferry parameters. Finally, we discuss the utility and limitations of the sticky Rouse approach for studying vitrimer viscoelasticity, and best practices for measuring $\eta_{0}$ and $\tau^{*}$. 


\section{Introduction}

Vitrimers are covalently cross-linked polymer networks that are insoluble in a good solvent, yet still flow at elevated temperatures. ${ }^{1-5}$ These paradoxical traits - a combination not found in other types of polymers - are enabled by their cross-links, which engage in thermoactivated associative exchange reactions that cause the network topology to fluctuate. In contrast to networks with dissociative cross-links, whose cross-link (XL) density follows an equilibrium relationship with temperature and concentration, ${ }^{3,6,7}$ vitrimers maintain network connectivity and XL density at all times and temperatures below degradation conditions. ${ }^{1-5,8}$ Conversion of a polymer to a vitrimer imparts it with improved solvent resistance and mechanical strength (like a thermoset) but does not compromise its ability to be processed by extrusion or other conventional techniques (like a thermoplastic). ${ }^{9-17}$ This marriage between high-performance and processability inspires significant interest into vitrimer structure-property relationships and applications. ${ }^{2-5}$ Here, we demonstrate a generalized Rouse model approach for relating the molecular structure of a vitrimer and its corresponding linear viscoelasticity.

The current framework for interpreting vitrimer rheology originates from the seminal epoxy vitrimer studies of Montarnal, Leibler, et al. ${ }^{1,18-20}$ For these materials, epoxy networks featuring $\beta$-hydroxy esters were doped with metal or organic catalyst. While at room temperature the vitrimers behaved as classical thermosets, at elevated temperatures the $\beta$-hydroxy esters underwent transesterification, allowing the epoxies to fully relax stress but still remain insoluble. The transient relaxation modulus was described by a simple Maxwell exponential decay, while the zero-shear viscosity and terminal relaxation times followed an Arrhenius relationship with temperature. The apparent activation energies estimated from these rheological properties $\left(E_{a}^{\mathrm{rh}}\right)$ were consistent with the activation energy for transesterification of small molecule epoxy analogues $\left(E_{a}^{\mathrm{sm}}\right)$. Extrapolation of the Arrhenius relationship provided $T_{v}$, the temperature at which the vitrimer viscosity equals $10^{12} \mathrm{~Pa} \mathrm{~s}$ - an empirical threshold for processability. Alteration of catalyst type tuned $E_{a}^{\text {rh }}$, while variation of the epoxy network chemical composition changed the glass transition tempera- 
ture $\left(T_{g}\right)^{1,18-20}$

Following these initial studies, researchers have generally focused on two different strategies for modifying vitrimer flow and mechanical properties: (i) altering the cross-linker exchange reaction chemistry to tune $E_{a}^{\text {rh }}$ or (ii) varying the vitrimer backbone flexibility to change $T_{g}$. For the cross-linker, efforts over the past decade have created a vast library of externally and internally catalyzed associative exchange reactions that may be incorporated into vitrimer networks. Such chemical properties as cross-linker structure, ${ }^{21-26}$ reactive functional group stoichiometry, ${ }^{17,27-31}$ catalyst loading, ${ }^{1,17,18,32}$ catalyst $\mathrm{pK}_{a},{ }^{33}$ and even coordination between cross-linker and catalyst modulate the $E_{a}^{\text {rh }}$ and rheological profile. ${ }^{34}$ For the backbone, the vitrimer concept has been adapted to a wide variety of commodity polymers, including those with high $T_{g},{ }^{9,10,12,16}$ moderate $T_{g},{ }^{27,35,36}$ low $T_{g},{ }^{21,29,37-39}$ or semi-crystallinity. ${ }^{40-42}$ Inclusion of branching, ${ }^{43-46}$ macro/microphase separation, ${ }^{12,13,47-49}$ or additives within the vitrimer matrix offers additional design parameters. ${ }^{50-53}$

While the synthetic toolset for vitrimers has grown quite sophisticated, understanding vitrimer thermorheological properties remains primitive. Vitrimers that exhibit an Arrhenius temperature dependence generally express an $E_{a}^{\mathrm{rh}}$ that is larger than $E_{a}^{\mathrm{sm}}$. Röttger et al. found that the $E_{a}^{\mathrm{rh}}$ of poly(methyl methacrylate) vitrimers with dioxaborolane XLs was $\approx 40-80 \mathrm{~kJ} / \mathrm{mol}$, much larger than the $E_{a}^{\mathrm{sm}}=15-30 \mathrm{~kJ} / \mathrm{mol}$ observed for small molecule dioxaborolanes undergoing metathesis. ${ }^{9,22}$ Lessard et al. and Spiesschaert et al. demonstrated that the ratio of $E_{a}^{\text {rh }}$ to $E_{a}^{\mathrm{sm}}$ for vitrimers with vinylogous urethane XLs is a function of the backbone chemistry. ${ }^{15,16,54}$ In this work, we hypothesize that the difference between $E_{a}^{\mathrm{rh}}$ and $E_{a}^{\mathrm{sm}}$ is related to the temperature dependence of chain friction. Moreover, the stress relaxation of vitrimers near their $T_{g}$ deviates from the simple Maxwell model. ${ }^{2}$ At this temperature regime, secondary plateaus and peaks commonly appear in small amplitude oscillatory shear measurements, ${ }^{29,55,56}$ alluding to the presence of additional relaxation modes and timescales. The observed relationship between the XL density and terminal relaxation time also varies drastically across systems. ${ }^{38,54,57}$

These complexities of vitrimer flow behavior motivate several theoretical approaches for understanding the rheology. Terentjev et al. pioneered the development of microscopic constitutive equa- 
tions to describe vitrimer stress relaxation, creep, and uniaxial deformation. Their theories highlighted the strong influence of the cross-linker exchange kinetics on the material response. ${ }^{44,45,58-60}$ Qi et al. used finite element modeling to relate cross-linker exchange to the stress distribution in vitrimers during deformation and surface welding. ${ }^{61-66} \mathrm{Wu}$ et al., Jourdain et al., and Fang et al. employed time-temperature superposition to collapse rheological data into master curves. In these works, superposition was not achieved over the entire relaxation spectrum, hinting that the systems had multiple relaxation modes with differing timescales and temperature dependences. ${ }^{56,67,68}$ In addition to continuum methods, molecular dynamics (MD) and Monte Carlo (MC) simulations provide deep insight into the relationship between structure and flow. Although the wide range of timescales in vitrimer systems makes it difficult to use standard atomistic molecular simulations, Perego and Khabaz overcame this barrier by employing hybrid MD/MC simulations to study expansion and chain diffusivity around $T_{v}{ }^{69}$ Using coarse-grained MD, Sciortino et al. found that the macroscopic vitrimer viscosity is a reflection of both network topology and cross-linker exchange kinetics. ${ }^{70-72}$ Coarse-grained slip link modeling also offers a pathway to interrogate the interactions between backbone relaxations and transient cross-linking. ${ }^{73}$

On a broader scale, vitrimers can be considered to be a subset of dynamically cross-linked polymer networks, for which several rheological theories have been already developed. The history of characterizing these networks can be traced back to the transient network model of Green and Tobolsky. ${ }^{74}$ Inspired by the theory of rubber elasticity, they proposed this model for polymer melts in which entanglements were treated as temporary junctions that could break and reform spontaneously. ${ }^{74}$ The basic formalism of transient networks was extended, generalized, ${ }^{75,76}$ and specialized for physically cross-linked networks and associating polymers. ${ }^{77-79}$ For unentangled polymers, Baxandall demonstrated that at long timescales, the dynamics of reversibly cross-linked chains follow Rouse dynamics. ${ }^{80,81}$ Leibler, Rubinstein, et al. comprehensively fleshed out the gelation and dynamic properties of reversible networks for unentangled ("sticky Rouse") and entangled ("sticky reptation") chains using scaling theory as their primary tool. ${ }^{82-85}$ The resulting framework is quite powerful and precisely portrays the dynamics of many complex polymeric 
systems, including ionomers, ${ }^{86,87}$ supramolecular polymers, ${ }^{88-90}$ complex coacervates, ${ }^{91-93}$ and polymer-protein conjugates. ${ }^{94}$

In this work, we employ the sticky Rouse model to investigate the linear viscoelasticity of monodisperse unentangled vitrimer melts. We assume that the lifetime of a XL $\left(\tau_{x}\right)$ obeys an $\operatorname{Ar}-$ rhenius relation with activation energy $E_{a}^{\mathrm{sm}}$, and a prefactor that is proportional to the monomer relaxation time - a fairly standard assumption used in modeling the rheology of dynamic networks. ${ }^{84,85,95,96}$ We focus on fully developed networks beyond the gel point, where the sticky Rouse model is ideally suited. We employ both a generalized sticky Rouse model - labeled the inhomogeneous Rouse model (IHR) - and a simplified sticky Rouse model (SSR) that provides an approximate solution. The questions we seek to address are the following:

1. Under what conditions does vitrimer rheology follow an Arrhenius temperature dependence?

2. What is the relationship between $E_{a}^{\mathrm{rh}}$ and $E_{a}^{\mathrm{sm}}$ ? How does the molecular structure, backbone flexibility, and cross-linker chemistry affect this relationship?

3. What are the potential pitfalls of using approximate methods to determine the zero-shear viscosity $\left(\eta_{0}\right)$ and characteristic relaxation time $\left(\tau^{*}\right)$ in estimating $E_{a}^{\text {rh }}$ from Arrhenius plots?

4. When do the IHR and SSR converge and diverge? What are the relative merits of one over the other?

We use the IHR and SSR to simulate the linear viscoelasticity of model vitrimers and describe the interplay between network strand relaxation and XL exchange. As expected, the different temperature dependences of the chain friction and Arrhenius modes leads to a breakdown of time-temperature superposition. XL density, kinetics, and distribution control both $\eta_{0}$ and $\tau^{*}$. Furthermore, due to the form adopted for $\tau_{x}$, the relationship between $E_{a}^{\mathrm{rh}}$ and $E_{a}^{\mathrm{sm}}$ depends on the chain friction and temperature window explored. This framework explains the empirical observation of $E_{a}^{\mathrm{rh}}>E_{a}^{\mathrm{sm}}$ and contends that $E_{a}^{\mathrm{rh}}$ may be estimated based solely on knowledge of the $E_{a}^{\mathrm{sm}}$ and Williams-Landel-Ferry parameters of the backbone. The presence of a pre-exponential 
factor in the model also offers an avenue for evaluating the mobility and mechanism of exchange of XLs within a vitrimer matrix. These findings not only provide insight into fundamental vitrimer structure-viscoelasticity relationships, but also highlight the importance of using rigorous practices to determine $\eta_{0}, \tau^{*}$, and $E_{a}^{\mathrm{rh}}$ from rheological measurements.

\section{Methods}

As shown in figure 1a, we consider a bead-spring chain with $N$ beads, of which $N_{x}$ beads are sticky. For vitrimers, these sticky beads correspond to associative XLs. We focus on fully developed vitrimer networks of unentangled polymer melts whose XL density is above the gel point. Thus, $N \lesssim N_{e}$, where $N_{e}$ is the number of monomers in an entanglement strand, and $N_{x} \geq 2$, where $N_{x}$ is the average number of XLs per chain.

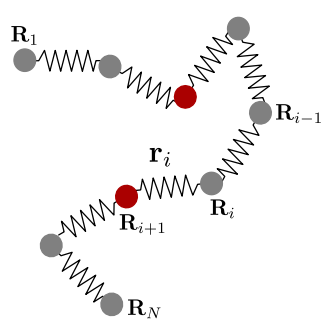

(a)

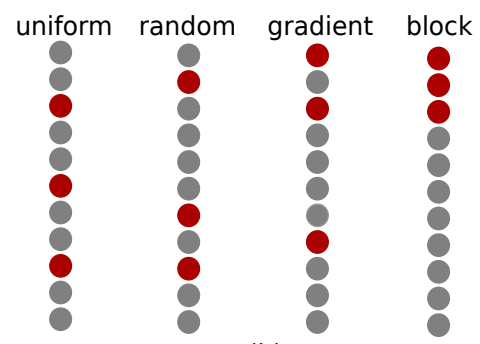

(b)

Figure 1: (a) Schematic diagram of a bead-spring chain with $N$ monomers, of which $N_{x}=2$ are sticky (red beads). (b) Four different distributions of sticky beads are considered: uniform, random, gradient, and block (see descriptions in text). For each distribution type, the sample chains have $N=11$ and $N_{x}=3$.

\subsection{Standard Rouse Model}

For the standard Rouse model, the chain has $N-1$ springs with spring constant $k=3 k_{B} T / b^{2}$, where $k_{B}$ is Boltzmann's constant, and $b$ is the statistical segment length. The beads are located at $\mathbf{R}_{i}$, where $i=1,2, \cdots, N$. The spring end-to-end vectors $\mathbf{r}_{i}=\mathbf{R}_{i+1}-\mathbf{R}_{i}$ for $i=1,2, \cdots, N-1$.

The equation of motion is controlled by spring and Brownian forces. ${ }^{97,98}$ These can be cast as 
a coupled set of equations for the springs,

$$
\dot{\mathbf{r}}_{i}+\sum_{j=1}^{N-1} C_{i j} \mathbf{r}_{j}=\mathbf{f}_{i}^{B}, \quad i=1,2, \cdots, N-1,
$$

where $\mathbf{f}_{i}^{B}$ is the Brownian force that satisfies the fluctuation-dissipation theorem. The tridiagonal matrix $\mathbf{C}$ encodes the connectivity of the springs. For the standard or "homogeneous" Rouse model without any sticky beads $\left(N_{x}=0\right), C_{i j}=(k / \zeta) A_{i j}$, where

$$
A_{i j}= \begin{cases}2 & \text { if } i=j \\ -1 & \text { if } i=j \pm 1 \\ 0 & \text { otherwise. }\end{cases}
$$

The ratio of the bead friction and spring constant, $\tau_{b}=\zeta / k$, is a natural timescale associated with bead or monomer relaxation. The eigenvalues of the matrix $\mathbf{C}$ are inversely proportional to spectrum of relaxation times,

$$
\lambda_{i}=\frac{4 k}{\zeta} \sin ^{2}\left(\frac{i \pi}{2 N}\right), \quad i=1,2, \ldots, N-1 .
$$

The Rouse stress relaxation time is half the end-to-end vector decorrelation time because it is obtained from a quadratic function of the amplitude of the normal modes. ${ }^{99}$ Thus, $\tau_{i}=1 /\left(2 \lambda_{i}\right)$, which implies,

$$
\tau_{i}=\frac{\zeta}{8 k \sin ^{2}(i \pi / 2 N)}=\frac{\tau}{\sin ^{2}(i \pi / 2 N)}, \quad i=1,2, \ldots, N-1
$$

where $\tau=\tau_{b} / 8$ is the elementary Rouse timescale.

For $N \gg 1$, the relation $\sin x \approx x$ is invoked to obtain the approximate spectrum (denoted by "hat"),

$$
\hat{\tau}_{i}=\frac{\tau_{1}}{i^{2}}=\frac{\zeta}{2 \pi^{2} k}\left(\frac{N}{i}\right)^{2}, \quad i=1,2, \ldots, N-1
$$


where $\hat{\tau}_{1}=\hat{\tau} N^{2}$, and $\hat{\tau}=\tau_{b} /\left(2 \pi^{2}\right)$. Note that the true and approximate spectra are equal only for the slow modes $(i \ll N)$. In particular, $\tau_{1} \approx \hat{\tau}_{1}$, but the elementary timescale $\tau \neq \hat{\tau}$. Instead, $\tau / \hat{\tau}=\pi^{2} / 4>1$. In the limit of large times, slow modes dominate the stress response. The difference in the spectrum at short timescales does not materially affect the $G(t)$ calculation given by,

$$
\phi(t)=\frac{G(t)}{G_{0}}=\frac{1}{N} \sum_{j=1}^{N-1} e^{-t / \tau_{j}}
$$

with modulus $G_{0}=\rho R T / M_{0}$, where $\rho$ is the density of the polymer melt, $R$ is the universal gas constant, and $M_{0}$ is the molar mass associated with a bead. The zero-shear viscosity is given by,

$$
\eta_{0}=\int_{0}^{\infty} G(t) d t=\frac{G_{0}}{N} \sum_{i=1}^{N-1} \tau_{i}
$$

The timescale $\tau_{\eta}$ associated with $\eta_{0}$ is given by the ratio of the viscosity and modulus, $\tau_{\eta}=\eta_{0} / G_{0}$. From eqn $7, \tau_{\eta}$ can be interpreted as the average relaxation time. Due to the dispersion in Rouse relaxation times, it is biased towards slow modes. For the standard Rouse model, if we approximate $\tau_{i}=\hat{\tau}_{i}=\hat{\tau}(N / i)^{2}$, and consider the limit of large $N$,

$$
\tau_{\eta}^{R} \approx \frac{\hat{\tau} N^{2}}{N} \sum_{i=1}^{N-1} \frac{1}{i^{2}} \approx \frac{\tau_{b} N}{2 \pi^{2}}\left(\frac{\pi^{2}}{6}\right)=\frac{N \tau_{b}}{12}
$$

This reflects the classic $\eta_{0} \sim N$ dependence, which is indeed observed empirically for short unentangled polymer melts. Note that $\tau_{\eta}^{R}$, which represents an average over all the timescales in the Rouse spectrum, is linear in $N$, while the longest relaxation time $\tau_{1} \sim N^{2}$. Depending on the use case, the characteristic relaxation time $\tau^{*}$ may be identified with either $\tau_{\eta}$ or $\tau_{1}$. Experimentally, $\tau_{\eta}$ is obtained simply from the ratio of the viscosity and modulus, while $\tau_{1}$ has to be calculated by fitting a discrete relaxation spectrum to the data. ${ }^{100-104}$

For convenience, the notation used for different timescales considered in this paper is summarized in Supporting Information Table S1. We prefer to use $\tau_{b}=\zeta / k$ as the fundamental unit of time to avoid the ambiguity that arises from different definitions of the elementary Rouse time ( $\tau$ 
or $\hat{\tau}$ ). Departures from this norm are indicated and justified wherever they occur.

\subsection{Sticky Rouse Model}

For the sticky Rouse model, the key idea is to incorporate the lifetime of a XL by increasing the frictional drag associated with sticky beads. Thus, the terms XL and sticky bead are used interchangeably in this paper. Furthermore, we assume that XLs are fully saturated, i.e., there are no unpaired sticky beads.

Unlike many dynamically cross-linked systems where the XL density changes as a function of temperature or concentration, for vitrimers the total number of XLs is strictly conserved. Dynamical changes in network structure are mediated by exchange reactions, rather than breakage and reformation of XLs. Due to the symmetry of XL exchange reactions, and the assumption of saturation of XLs, the number and location of sticky beads on participating chains is preserved. This makes the sticky Rouse model particularly well-suited. Furthermore, since the number of XLs is baked in at synthesis, $N_{x}$ is independent of temperature. We entertain two different versions of the sticky Rouse model: the inhomogeneous Rouse (IHR) model and the simplified sticky Rouse (SSR) model. The IHR is fine-grained at the level of a Kuhn segment, and can readily account for changes in viscoelastic properties due to non-uniform distribution of XLs.

\subsubsection{Inhomogeneous Rouse Model}

The IHR model is a generalization of the standard or homogeneous Rouse model. It relaxes the constraint of uniform spring constants and drag coefficients; therefore, $k_{i} \neq k$ and $\zeta_{i} \neq \zeta$, where $k_{i}$ is the spring constant of the $i^{\text {th }}$ spring, and $\zeta_{i}$ is the drag coefficient of the $i^{\text {th }}$ bead. This model was initially proposed by three different groups, nearly simultaneously, to predict the linear rheology of amorphous mixtures of block polymers. ${ }^{105-107}$ In these studies, the difference between the two blocks was represented as a difference in friction coefficients. Similar versions of the theory were also used to study bidisperse homopolymer melts with short and long chains, ${ }^{108}$ analyze chain dynamics near the glass transition temperature, ${ }^{109}$ and to investigate the eigenmodes of relaxation 
in associating polymers by invoking the idea of sticky Rouse beads. ${ }^{110}$

In the IHR model, the equation of motion is still governed by eqn 1, albeit with a more general tridiagonal matrix $\mathbf{C}$. For $i=1,2, \ldots, N-1$, the nonzero elements of $\mathbf{C}$ are given by,

$$
\begin{aligned}
C_{i, i-1} & =-k_{i-1} / \zeta_{i} \\
C_{i, i} & =k_{i} / \zeta_{i}+k_{i} / \zeta_{i+1} \\
C_{i, i+1} & =-k_{i+1} / \zeta_{i+1},
\end{aligned}
$$

assuming $k_{0}=k_{N}=0$, in these expressions. In this work, we set $k_{i}=k=3 k_{B} T / b^{2}$, assuming that all the beads (regular or sticky) are separated by the same average distance $b$. The frictional drag associated with the $N_{x}$ sticky beads is denoted by $\zeta_{x}$; similarly the drag associated with the remaining $N-N_{x}$ regular beads is denoted by $\zeta$. The eigenvalues of $\mathbf{C}$ can be numerically evaluated to obtain the spectrum of $N-1$ relaxation times, $\tau_{i}=1 /\left(2 \lambda_{i}\right),{ }^{110,111}$ from which the stress relaxation response can be obtained using eqn. 6 .

\subsubsection{Simplified Sticky Rouse Model}

For the simplified sticky Rouse (SSR) model, an approximate solution is obtained when the sticky and regular Rouse modes are well-separated $\left(\zeta_{x} \gg \zeta\right)$, and the number of XLs/chain is sufficiently large $\left(N_{x} \gg 1\right)$. The SSR model asserts that the stress relaxation of unentangled monodisperse associating polymers contains two sets of non-interacting Rouse-like contributions, ${ }^{80,82,86,112}$

$$
\phi(t)=\frac{G(t)}{G_{0}}=\frac{1}{N}\left[\sum_{j=1}^{N_{x}-1} \exp \left(-\frac{j^{2} t}{\tau_{x} N_{x}^{2}}\right)+\sum_{j=N_{x}}^{N-1} \exp \left(-\frac{j^{2} t}{\tau N^{2}}\right)\right] .
$$

The first summation contains slow sticky modes that arise due to XL exchange, and the second summation includes fast Rouse modes of the underlying polymer chain. Here, the timescales $\tau_{x}$ and $\tau$ are related to the viscous drag, $\zeta_{x}$ and $\zeta$, associated with the sticky and regular Rouse beads (figure 1a), respectively. As demonstrated in sec. 3.1, under certain conditions, the SSR model is 
an excellent approximation to the IHR model.

It is helpful to highlight the differences between the two versions of the sticky Rouse model considered here. Unlike the SSR model, the IHR model does not regard the regular and sticky modes as independent contributions that can be summed up. Instead, it considers a single Rouse chain where the slow and fast modes are free to interact. The expression for the Rouse time $\tau_{x} N_{x}^{2}$, corresponding to the XLs in the SSR, implicitly assumes $N_{x} \gg 1$, which may not be

true for lightly cross-linked chains. The $N \gg 1$ approximation inherent in the SSR is valid for systems studied here so that artifacts that arise due to small number of effective segments are negligible. Nevertheless, caution should be exercised for low molecular weight polymers with stiff backbones, where this assumption may be questionable. The IHR does not directly invoke this approximation, and is therefore less susceptible to this problem. Furthermore, the response of the IHR can account for different distributions of sticky beads along the chain. As shown later, when $N_{x} \gg 1, \tau_{x} N_{x}^{2} \gg \tau N^{2}$, and the XLs are distributed uniformly or randomly, the IHR and SSR models agree with each other. Thus, the SSR can be thought of as a special case of the IHR, which is convenient to use under appropriate conditions.

\subsection{Distribution of XLs}

Recent advances in polymer chemistry have opened the door for synthesizing vitrimers with a variety of different microstructures. ${ }^{2}$ Thus, it is interesting to examine how XL distribution throughout the vitrimer network affects viscoelasticity. Here we consider four different distributions of sticky beads, as depicted schematically in fig. 1b: (i) uniform, (ii) random, (iii) gradient, and (iv) block. Note that we do not account for composition fluctuations or microphase separation, essentially assuming that the $\chi$-parameter characterizing the enthalpic interaction between regular and sticky beads is zero.

For uniform distribution, we determine the spacing $\Delta N=(N+1) /\left(N_{x}+1\right)$. When possible, $N$ and $N_{x}$ are selected so that $\Delta N$ is an integer; otherwise $\Delta N$ is rounded to the nearest integer. The probability $\rho_{x}^{i}$ of marking bead $i$ as sticky is assumed to be $\rho_{x}^{i}=1$ when $i / \Delta N$ is an integer, 
and zero otherwise. In the example shown in fig. $1 \mathrm{~b}, N=11$ and $N_{x}=3$; thus, $\Delta N=(11+$ 1) $/(3+1)=3$, and beads 3, 6 , and 9 are marked as sticky beads. This protocol is deterministic: a particular choice of $N$ and $N_{x}$ uniquely determines the architecture of the sticky Rouse chain.

For random distribution, we consider each bead along the chain in turn. With a constant probability $\rho_{x}=N_{x} / N$, it is marked as a sticky bead; otherwise it is marked as a regular bead. Since this protocol is stochastic, the number of XLs on any particular chain $n_{x}$ may deviate from the prescribed $N_{x}$, and is binomially distributed,

$$
\pi\left(n_{x} ; \rho_{x}, N\right)=\left(\begin{array}{c}
N \\
n_{x}
\end{array}\right) \rho_{x}^{n_{x}}\left(1-\rho_{x}\right)^{N-n_{x}}
$$

For the random distribution of XLs, we average the response over an ensemble of 1000 chains.

For gradient distribution, the probability $\rho_{x}^{i}$ is not constant. Instead, it increases from one end to the other. Thus, we set $\rho_{x}^{1}=1$, and $\rho_{x}^{N}=0$. For internal beads, we assume a form,

$$
\rho_{x}^{i}=\left(\frac{N-i}{N-1}\right)^{\alpha}
$$

where $\alpha$ is determined by requiring the average or expected number of XLs per chain to equal $N_{x}$,

$$
\sum_{i=1}^{N} \rho_{x}^{i}=\sum_{i=1}^{N}\left(\frac{N-i}{N-1}\right)^{\alpha}=N_{x}
$$

The value of $\alpha$ is determined numerically, and for large $N$ and $N_{x}$, it is usually close to $\alpha \approx \rho_{x}^{-1}-1$. Note that the method is stochastic, and like random distributions, we average the response over an ensemble of chains.

Block distribution can be thought of as an extreme case of gradient distribution, where all the sticky beads are concentrated at one end. Therefore, $\rho_{x}^{i}=1$ for $i=1, \cdots N_{x}$, and $\rho_{x}^{i}=$ 0 , for $N_{x}<i \leq N$. Block and uniform distributions are deterministic, while the other two distributions are not. One can think of random distribution as a stochastic perturbation of the uniform distribution. Similarly, one can think of gradient distribution as intermediate between 
random and block distributions.

\subsection{Cross-link Frictional Drag}

Since the lifetime of a XL is governed by a chemical reaction, it follows an Arrhenius relation, ${ }^{113}$

$$
\tau_{x}=\tau_{x}^{0} e^{E_{a}^{\mathrm{sm}} / R T}
$$

where $E_{a}^{\mathrm{sm}}$ is the activation energy for the chemical reaction, and $\tau_{x}^{0}$ is the pre-exponential factor. In the homogeneous Rouse model the timescale $\tau \sim \zeta / k$. Since $k \sim k_{B} T / b^{2}$ is assumed to be uniform, it implies $\zeta \sim \tau$ and $\zeta_{x} \sim \tau_{x}$. However, based on scaling arguments for the self-diffusivity of chains, we can derive a more general expression for the usual scenario where $\tau_{x}>\tau$.

First, we recap the argument presented by Colby et al. ${ }^{95}$ The diffusivity $D_{0}$ of a Rouse chain without XLs $\left(N_{x}=0\right)$ with relaxation time $\mathcal{T}_{0} \sim \tau N^{2}$ and dimensions $R^{2} \sim N b^{2}$ is,

$$
D_{0} \sim \frac{R^{2}}{\mathcal{T}_{0}}=\frac{N b^{2}}{\tau N^{2}}=\frac{b^{2}}{\tau} \frac{1}{N}
$$

For a sticky Rouse chain with $\rho_{x} \ll 1$, we can crudely approximate $\mathcal{T} \approx \tau_{x} N_{x}^{2}+\tau N^{2}$ so that it is governed by the slowest (regular or sticky) Rouse mode. If the sticky modes dominate the late-time response, $\tau_{x} N_{x}^{2} \gg \tau N^{2}$, then $\mathcal{T} \approx \tau_{x} N_{x}^{2}$, and,

$$
D \sim \frac{R^{2}}{\mathcal{T}}=\frac{N b^{2}}{\tau_{x} N_{x}^{2}}=\frac{b^{2}}{\tau_{x}} \frac{N}{N_{x}^{2}} .
$$

Note that this argument implies $D \sim N_{x}^{-2}$, which is empirically observed for unentangled, lightly sulfonated polystyrene ionomers. ${ }^{95}$ In general, however, the ratio of the diffusivities,

$$
\frac{D_{0}}{D}=1+\frac{\tau_{x} N_{x}^{2}}{\tau N^{2}}
$$

For free-draining chains, we can obtain an expression for the ratio of the diffusivities in terms 
of the drag coefficient, using the Einstein relation. For a plain Rouse chain without any XLs, $D_{0}=k_{B} T /(\zeta N)$, which is identical to eqn. 15 with $\zeta=k \tau$. For a sticky Rouse chain, the total drag may be written as $\zeta_{x} N_{x}+\zeta\left(N-N_{x}\right)$. The ratio of the diffusivities,

$$
\frac{D_{0}}{D}=1+\frac{\left(\zeta_{x}-\zeta\right) N_{x}}{\zeta N}
$$

Comparing eqns 17 and 18, we find the ratio of the drag coefficients $\delta$, corresponding to sticky and regular beads, is approximately proportional to the product of the $\tau_{x}$ and $N_{x}$,

$$
\delta=\frac{\zeta_{x}}{\zeta}=1+\frac{\tau_{x}}{\tau} \frac{N_{x}}{N}
$$

When slow modes dominate the response, i.e., $\tau_{x} N_{x} \gg \tau N, \delta$ is proportional to the product of $\tau_{x}$ and $N_{x}$ because $\delta \approx \tau_{x} N_{x} /(\tau N)$. As such, we obtain the anticipated $\zeta_{x} \sim \tau_{x}$ relation. In the opposite extreme, when the chain is lightly cross-linked $\left(\rho_{x} \ll 1\right)$ and the lifetime of a XL is short $\left(\tau_{x} \sim \tau\right), \delta \approx 1$

\subsection{Temperature Dependence}

The pre-exponential factor $\tau_{x}^{0}$ in eqn 14 subsumes a lot of interesting and ill-understood physics. This is true even for interpreting chemical reaction experiments of non-polymeric systems. Over limited temperature windows, $\tau_{x}^{0}$ is often assumed to be independent of temperature as its variability is typically dwarfed by the exponential term. However, this assumption of constancy can lead to serious errors (of the order of $10-50 \%$ ) in estimating the activation energy from small molecule studies, especially when $E_{a} / R T \lesssim 10 .{ }^{114}$ When more precise analyses are required, especially when experiments are performed over a wide temperature range, $\tau_{x}^{0}$ is modeled as a function of temperature. For polymers, a fundamental timescale is set by monomer friction. As such, it is perhaps natural to propose $\tau_{x}^{0} \sim \tau$, where $\tau \sim \zeta / k$ is the elementary Rouse timescale. We set $\tau_{x}^{0}=2 \tau$ to account for the decreased mobility of a XL bead which is connected to four strands, instead of two. ${ }^{115}$ We discuss the repercussions of this assumption in sec. 4.1. 
Above the glass transition temperature $T_{g}$, the temperature dependence of $\tau$ for polymers and networks is empirically described by the Williams-Landel-Ferry (WLF) equation, ${ }^{116}$

$$
\log _{10} \alpha_{T}=\frac{-C_{1}\left(T-T_{0}\right)}{C_{2}+\left(T-T_{0}\right)}
$$

where $T_{0}$ is the temperature at which $\alpha_{T}=1$, and $C_{1}$ and $C_{2}$ are parameters. For $T \approx T_{g}$, $\alpha_{T}$ is usually very sensitive to temperature. However, at sufficiently high temperatures, where $T \gg T_{\infty} \equiv T_{0}-C_{2}, \alpha_{T}$ becomes less sensitive to temperature. In this regime, the WLF equation can be described approximately using an Arrhenius form, ${ }^{117}$

$$
\alpha_{T} \approx \exp \left(\frac{E_{\mathrm{WLF}}}{R T}\right) \quad \text { with } \quad \frac{E_{\mathrm{WLF}}}{R T}=\frac{2.303 C_{1} C_{2} T}{\left(C_{2}+T-T_{0}\right)^{2}}
$$

With this choice for $\tau_{x}^{0}$, we anticipate acceleration of dynamics with increased temperature. Since $\tau_{x}$ is a product of a WLF and an Arrhenius term, its temperature dependence is stronger than both the WLF and Arrhenius contributions. This trend is indeed observed in ionomers, where the formation and disassociation of XLs is governed by electrostatic interactions and dielectric contrast. ${ }^{56}$ WLF parameters may be perturbed when chains are chemically modified to enable cross-linking; however, this perturbation is neglected in this work.

The modulus $G_{0}$ also varies with temperature, although it exhibits a somewhat weaker dependence. This change is often described using a vertical shift factor,

$$
b_{T}=\frac{\rho(T) T}{\rho\left(T_{r}\right) T_{r}}
$$

where $T_{r}$ is any convenient reference temperature. The WLF equation can be shifted to the reference temperature $T_{r}$, by defining,

$$
a_{T}=\frac{\alpha_{T}(T)}{\alpha_{T}\left(T_{r}\right)}
$$

so that $a_{T}\left(T_{r}\right)=1$, instead of the default $\alpha_{T}\left(T_{0}\right)=1$. 


\section{Results}

We first compare the IHR and SSR models to delineate the regime in which the latter is a useful approximation. We then probe the general properties of the IHR model, including the influence of XL distribution on viscoelasticity, which cannot be studied with the SSR model. We use the IHR model to study the temperature dependence of viscoelasticity for three different polymer matrices. We find that time-temperature superposition cannot be performed across the entire time spectrum using a single set of shift parameters. We finally investigate the temperature dependence of viscosity on the terminal relaxation time in these systems, and offer guidelines for ensuring accurate measurement of these parameters.

\subsection{Comparison of IHR and SSR Models}

Figure 2 compares the IHR and SSR models for different values of $\tau_{x}$ and $N_{x}$. We assume that $N=119, \tau_{b}=\zeta / k=1$, and that the XLs are distributed uniformly along the chain. As $\tau_{x} / \tau$ increases from $10^{2}$ to $10^{3}$, the "stickiness" of the XLs also increases. Regardless of this ratio, the correspondence between the IHR and SSR in the limit of $N_{x}=0$ and $N_{x}=N$ is quite good. In these two limiting cases, only one of the two summations in eqn 10 describing the SSR model is operative. The response is effectively Rouse-like, which is evident in figure 2 . The vertical dotted lines denote the longest relaxation times $\left(\tau_{1}\right)$ of the bare chain and fully sticky chain $-\tau N^{2}$ and $\tau_{x} N^{2}$, respectively. For the two limiting cases, $\delta=1$ when $N_{x}=0$, and $\delta \approx \tau_{x} / \tau\left(10^{2}\right.$ or $\left.10^{3}\right)$ when $N_{x}=N$. Note that we use the ratio $\tau_{x} / \tau$ instead $\tau_{x} / \tau_{b}$ to characterize the relative stickiness of XLs because it serves as a convenient proxy for $\delta$. It is also a natural choice in the SSR due to the form of eqn 10, and is used in figures 2 and 3 where comparisons to IHR are made.

Figure 2 also illustrates the response for $N_{x}=19$, where only some of the beads are sticky, rather than all or none. Its stress relaxation response lies between these two extremes. Values of $N_{x}$ and $N$ are chosen so that the number of beads between the uniformly spaced XLs is an integer. When $\tau_{x} / \tau=10^{3}(\delta \approx 160)$, the agreement between the two models is still good because the sticky 

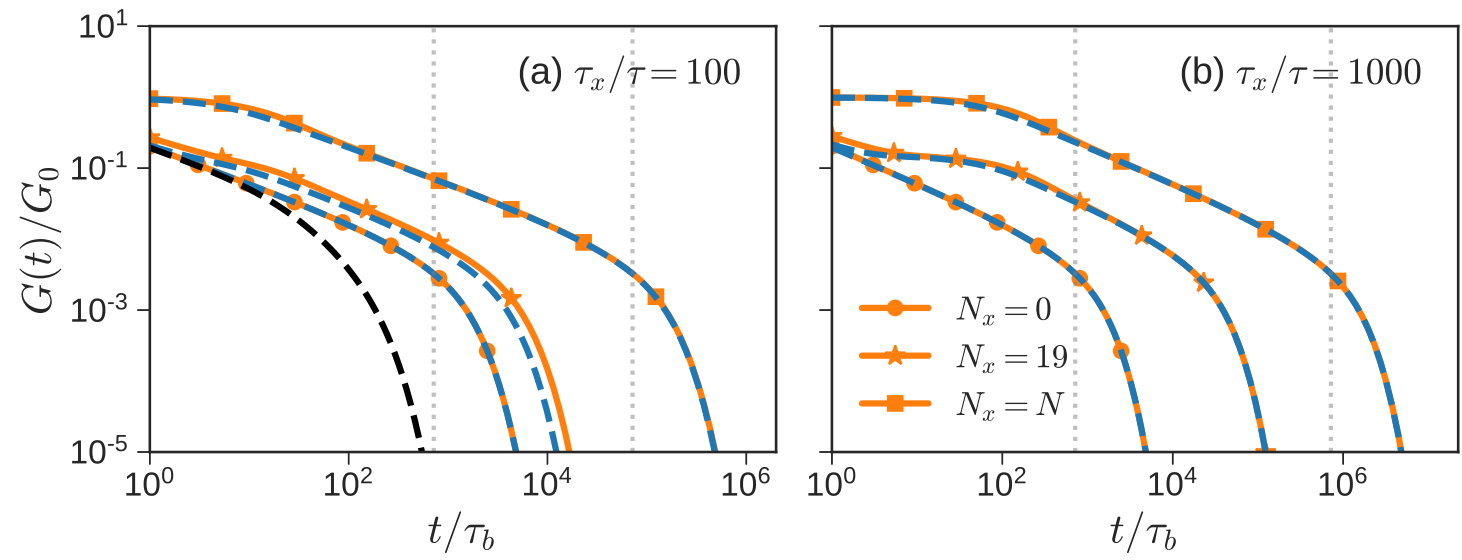

Figure 2: Comparison between SSR (dashed lines) and IHR (solid lines with symbols) models for a chain with $N=119$ and varying values of $N_{x}$ (marked by different symbols). The vertical dotted lines indicate the longest relaxation times $\left(\tau_{1}\right)$ of the bare chain $\left(N_{x}=0\right)$ and the fully sticky chain $\left(N_{x}=N\right)$. For $N_{x}>\sqrt{\tau / \tau_{x}} N$, the correspondence between the two models improves, as the stickiness of the XLs increases. (a) $\tau / \tau_{x}=100$. The dashed black line shows the SSR response when $N_{x}=4$, which is less than the critical threshold of $\sqrt{\tau / \tau_{x}} N$. (b) $\tau / \tau_{x}=1000$.

and regular Rouse modes are well-separated, i.e., $\tau_{x} N_{x}^{2} / \tau N^{2} \approx 25$. In contrast, when $\tau_{x} / \tau=10^{2}$ $(\delta \approx 16), \tau_{x} N_{x}^{2} / \tau N^{2} \approx 2.5$, creating deviations between the two models that appear in figure $2 \mathrm{a}$. In the case that the XL density is equal to or less than the critical limit $\rho_{x}=N_{x} / N \lesssim \sqrt{\tau / \tau_{x}}$, violation of the $\tau_{x} N_{x}^{2} \gg \tau N^{2}$ assumption causes the SSR model to fail. When $\tau_{x} / \tau=10^{2}$, a value of $N_{x}<N / 10 \approx 12$ generates a physically incorrect response. In figure $2 \mathrm{a}$, for example, the dashed black line shows the response for $N_{x}=4$. Despite the presence of sticky beads, this response decays faster than the bare Rouse chain.

Figure 3 plots the average relaxation time $\tau_{\eta}=\eta_{0} / G_{0}$, which more clearly demonstrates the failure of the SSR model. Here, $\tau_{\eta}$ is estimated using the IHR and SSR for $N=119$ at $\tau_{x} / \tau=$ $10^{1}-10^{3}$ and as $N_{x}$ is systematically increased. The $\tau_{x} / \tau=10^{2}$ and $10^{3}$ curves are shifted up by one and two decades, from $\tau_{\eta}^{R} / \tau_{b} \approx 10$ to $\approx 100$ and $\approx 1000$, respectively, to reduce visual clutter. At $N_{x}=0$, both the IHR and SSR reduce to the bare Rouse chain, and the curves for all $\tau_{x} / \tau$ values coincide at $\tau_{\eta}^{R} / \tau_{b}=N / 12 \approx 10$ (see eqn 8 ). As $N_{x}$ increases, the IHR predicts a monotonic increase in $\tau_{\eta}$. Conversely, the SSR predicts an unphysical non-monotonic behavior. 
The $\tau_{\eta}$ obtained from the SSR decreases initially as $N_{x}$ increases, reaches a minima, and then increases to catch up with the IHR result. This is most clearly visible at $\tau_{x} / \tau=10^{1}$, but is present in all cases. As alluded to previously, this behavior is observed when the criterion $\tau_{x} N_{x}^{2} \gg \tau N^{2}$ is violated; in this regime, the SSR is not expected to work.

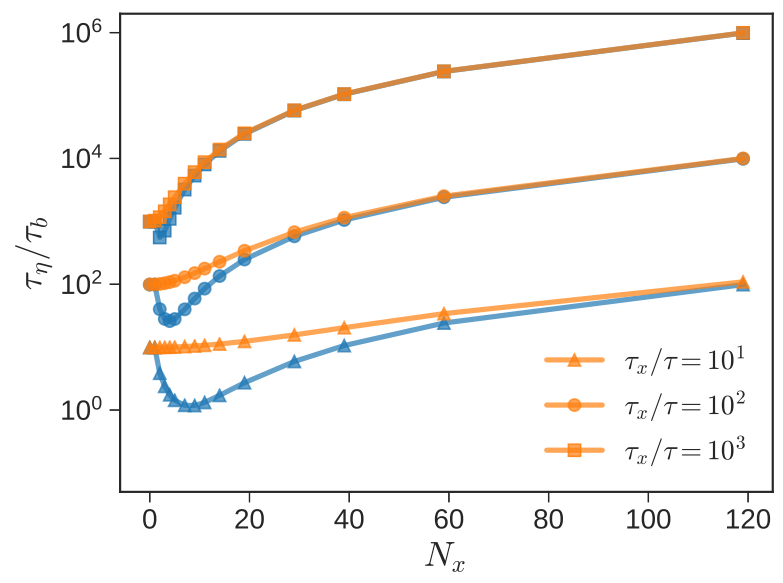

Figure 3: The characteristic relaxation time $\tau_{\eta}=\eta_{0} / G_{0}$ for the IHR (orange) and SSR (blue) models at $\tau_{x} / \tau=10^{1}$ (triangles), $10^{2}$ (circles), and $10^{3}$ (squares) as a function of the number of XLs for a chain with $N=119$ beads. XLs are distributed uniformly. The $\tau_{x} / \tau=10^{2}$ and $\tau_{x} / \tau=10^{3}$ curves are shifted upwards by a factor of 10 and 100 , respectively, for improved visibility.

To summarize, when $\tau_{x} N_{x}^{2} \gg \tau N^{2}$ and $N_{x} \gg 1$, the IHR and SSR models agree with each other. In this regime, it is perhaps preferable to use the SSR model due to its simplicity. This condition may be violated for chains with very few XLs (small $N_{x}$ ), or when the activation energy corresponding to the exchange reaction is relatively small. The latter is the case for dioxaborolane metathesis and imine exchange reactions, where $E_{a}^{\mathrm{sm}} \approx 10-30 \mathrm{~kJ} / \mathrm{mol}$ was measured from small molecule analogues. ${ }^{22}$ Under these circumstances, the use of the more general IHR model is advised. It has the advantage of being able to distinguish between different distributions of sticky beads, and remains valid even when $\tau_{x}$ and $N_{x}$ are small. 

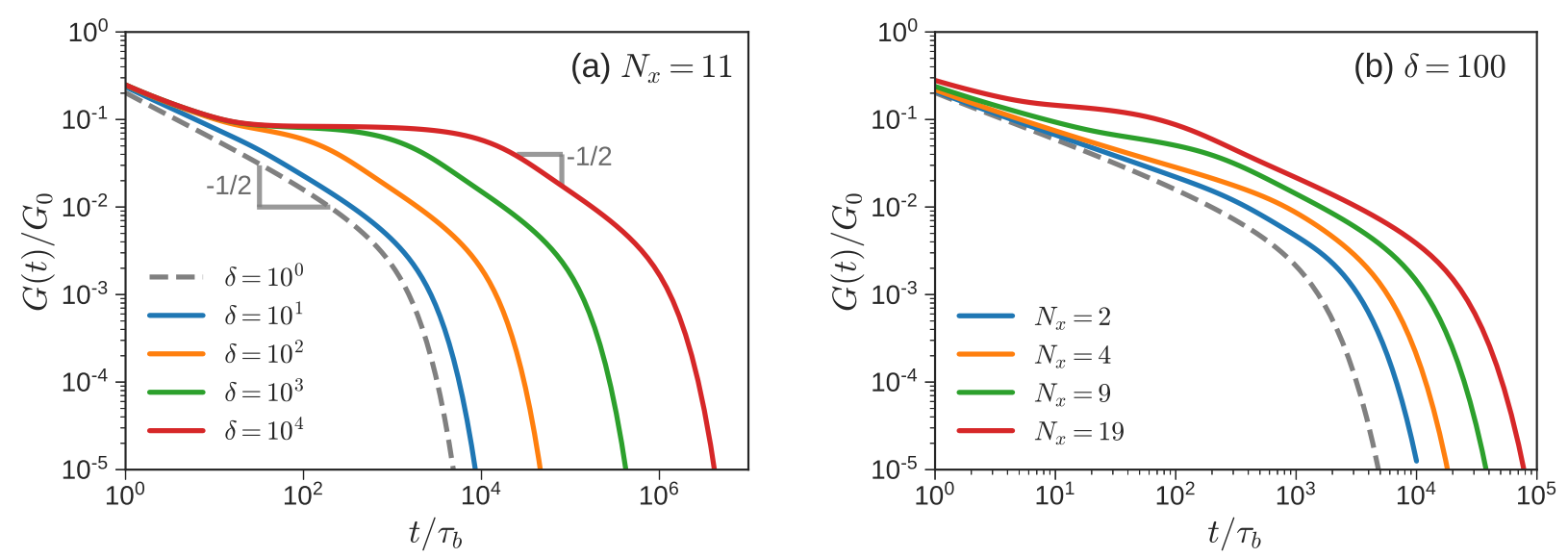

Figure 4: Normalized stress relaxation modulus for vitrimers with uniformly distributed XLs, as calculated by the IHR model. $G(t)$ is presented for varying (a) stickiness $\delta=\zeta_{x} / \zeta$ and (b) number of XLs $N_{x}$. Dashed line depicts the response of the bare Rouse chain with $N=119$. For (a), relaxation dynamics are retarded as $\delta$ is increased. The slopes of $-1 / 2$ corresponding to the bare and sticky Rouse modes are identified. For (b), increasing $N_{x}$ increases the terminal relaxation time and the plateau modulus.

\subsection{Properties of IHR Model}

Since the IHR model is both more general and robust than the SSR model, all results presented henceforth in this work are obtained from it. As our base case, we consider once again a chain with a total of $N=119$ monomers. XLs are uniformly distributed along the backbone, and values of $N_{x}$ are chosen so that the spacing between XLs is an integer. This choice ensures that the IHR model calculations are not jagged, and are free from artifacts that arise due to rounding or truncation.

Figure 4a portrays the change in $G(t)$ as the stickiness of the beads $\delta=\zeta_{x} / \zeta$ is increased from 1 to $10^{4}$. Here, $N_{x}=11$ so that $\Delta N=10$. The dashed gray line represents the case where $\delta=1$ and $\zeta_{x}=\zeta$, which is equivalent to the response of the bare Rouse chain. When $\delta$ is increased to 10 , the dynamics of the chain are retarded, and the $G(t)$ curve shifts rightward. As $\delta$ is increased further the response becomes slower. For $\delta>1$, the initial decrease in the modulus deviates from the $G(t) \sim t^{-1 / 2}$ trend of the bare Rouse chain. For sufficiently large $\delta \sim 10^{3}-10^{4}$, the plateau associated with the XLs becomes conspicuous. The height of this plateau $G_{x}=\rho_{x} G_{0}$ is independent of $\delta$. The terminal relaxation associated with XL exchange reactions also follows a 
Rouse-like pattern; beyond the plateau, we observe a second characteristic $G(t) \sim t^{-1 / 2}$ regime, before complete relaxation at approximately $\tau_{x} N_{x}^{2}$. For large $\delta$ and fixed $\rho_{x}=N_{x} / N$, eqn 19 implies that $\tau_{x}$ is proportional to $\delta$. In this regime $\left(\delta \gtrsim 10^{2}\right)$, the average relaxation time $\tau_{\eta} \sim \delta$. As $\delta$ increases by a factor of $10, \tau_{\eta}$ and $\eta_{0}$ also increase by a factor of 10 .

Figure $4 \mathrm{~b}$ depicts the variation of the modulus with the number of XLs. As in figure $4 \mathrm{a}$, we consider a chain with $N=119$ and uniformly distributed XLs. Here $\delta=100$ is held fixed. The dashed line corresponding to the bare Rouse chain is indeed the same in both subfigures. As $N_{x}$ increases from 2 to 19 , the number of monomers between successive XLs falls from 40 to 6 . Unlike $\delta$, increasing $N_{x}$ affects both $G_{x}$ and $\tau_{\eta}$. $G_{x}$ increases with increasing $N_{x}$ because it is proportional to $\rho_{x} . \tau_{\eta}$ increases with the number of XLs, becoming approximately proportional $\tau_{\eta} \sim N_{x}$ for $N_{x} \gg 1$. This can be understood through the standard Rouse model dependence of viscosity on molecular weight, or eqn 8 , where $N$ and $\tau_{b}$ are replaced by the number of sticky Rouse beads $N_{x}$ and their lifetime $\tau_{x}$, respectively.

Figure 5 focuses on the effect of the XL distribution. In figure 5a, we consider uniform and random distributions for a chain with $N_{x}=4,14$, and 29 XLs. For the random distribution, we report an average over 1000 independent replicas. At a given value of $N_{x}$, the stress response of the two distributions is effectively the same. Unlike the IHR, the SSR model cannot directly account for the impact of the distribution of the XLs on the dynamics. However, in the large $N_{x}$ regime the insensitivity to random or uniform distribution suggests that the SSR is a reasonable model to compute the linear viscoelasticity of random or uniformly distributed XLs.

Figure $5 \mathrm{~b}$ concentrates on $N_{x}=29$, and compares the response of the gradient and block distributions to the uniform and random distributions shown previously. Due to its stochastic nature, the response of the gradient distribution is averaged over 1000 independent replicas, just like random distribution. It shows significantly faster relaxation than random or uniform distributions. This is further exaggerated for block distributions, in which sticky beads are completely sequestered to one of the chain ends. Qualitatively, these observations are general, and persist for a broad range of values of $N, N_{x}$, and $\delta$. 

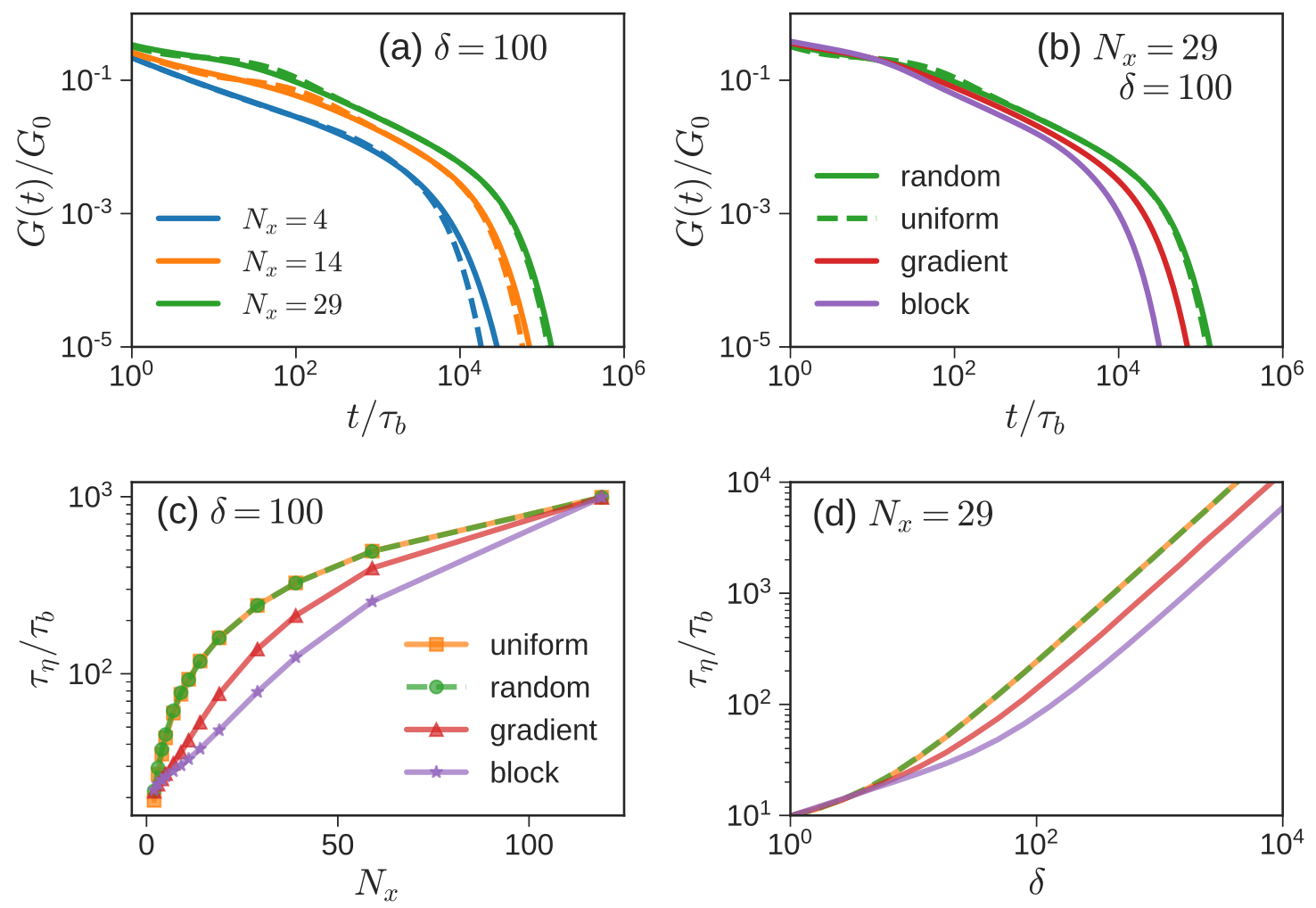

Figure 5: (a) Solid and dashed lines depict the stress relaxation response for randomly and uniformly distributed XLs, respectively, for a chain with $N=119$ and $\delta=100$. Three different values of $N_{x}=4,14$, and 29 are shown. Subfigure (b) focuses on one of these samples $\left(N_{x}=\right.$ 29), and compares the response of uniform and random XL distributions with blocky, and gradient distributions. The average relaxation time $\tau_{\eta}$ (proportional to $\eta_{0}$ ) is plotted as a function of (c) $N_{x}$ with $\delta=100$, and (d) $\delta$ with $N_{x}=29$. 
Figure 5c examines the average relaxation time $\tau_{\eta}$ as a function of distribution type. We consider chains with $N=119$ and $\delta=100$, and vary the number of XLs/chain. As expected, $\tau_{\eta}$ is independent of distribution type for the extreme cases of $N_{x}=0$ (bare Rouse chain) and $N_{x}=N$ (all beads are sticky). The difference between random and uniform distributions is barely perceptible over the entire range of $N_{x}$ explored. The gradient and block distributions follow trends that are anticipated from figure 5b. At a given $N_{x}$, as the distribution of XLs changes from even or approximately even (uniform/random) to concentrated at one of the ends approximately (gradient) or strictly (block), $\eta_{0}$ decreases.

Figure 5d varies $\delta$ between $10^{0}-10^{4}$, with $N_{x}=29$ held constant. For sufficiently large $\delta$ ( $\gtrsim 500), \tau_{\eta} \sim \delta$ is independent of the type of distribution. However, the relative order of viscosities (uniform $\approx$ random $>$ gradient $>$ block) is preserved. In this limit, the ratio of viscosities with different distributions becomes constant. At the other end, as $\delta$ approaches 1 and the sticky beads become less sticky, the four curves converge to the bare Rouse chain result.

We posit that the gradient and block XL distributions exhibit fast relaxations because they have a relatively long dangling chain end. Strong evidence for this pattern of relaxation is presented in the original paper on the retardation time spectrum of multiblock polymers. ${ }^{105}$ Besides other configurations, diblock $(\mathrm{AB})$ and triblock $(\mathrm{ABA}$ and $\mathrm{BAB})$ polymers were compared, where the "A" and "B" blocks correspond to sticky and regular beads, respectively. At a fixed concentration of sticky beads, it was found that relaxation was fastest (slowest) for BAB (ABA), where the sticky beads were confined near the core (near the ends) of the polymer. The relaxation of the corresponding diblock polymer was in between these two extremes.

To summarize, the IHR model can explore the linear viscoelastic response as a function of XL distribution. The linear rheology of uniform and random distributions of XLs are similar, and converge in the limit of large $N_{x}$. For uniformly distributed XLs, when $\delta \gtrsim 500$ and $N_{x} \gtrsim 20$ the IHR model predicts that the average relaxation time $\tau_{\eta}$ is approximately proportional to $\delta$ and $N_{x}$. The plateau associated with XLs, $G_{x}$, is independent of $\delta$ but proportional to $N_{x}$. At a given value of $\delta$ and $N_{x}$, the relative order of viscosities $\eta_{0}$ for different XL distributions is given by uniform 
$\approx$ random $>$ gradient $>$ block. This trend, however, is only applicable for homogeneous vitrimer systems where $\chi$-parameter between regular and sticky beads is zero.

Table 1: Selected properties of three common vitrimer matrices: polydimethylsiloxane (PDMS), polystyrene (PS), and poly(methyl methacrylate) (PMMA).

\begin{tabular}{lrrr} 
& PDMS & PS & PMMA \\
\hline$N_{e}$ & 165 & 127 & 100 \\
$M_{e}$ & 12293 & 13309 & 10013 \\
$T_{g}[\mathrm{~K}]$ & 150 & 373 & 407 \\
\hline \multicolumn{4}{c}{ WLF parameters } \\
\multicolumn{4}{c}{117,118} \\
$C_{1}$ & 1.9 & 12.7 & 9.4 \\
$C_{2}[\mathrm{~K}]$ & 222 & 50 & 447 \\
$T_{0}[\mathrm{~K}]$ & 303 & 373 & 463 \\
\hline
\end{tabular}

\subsection{Temperature Dependence of Viscosity}

We now use the IHR model to examine the thermorheological properties of model vitrimers. Table 1 shows the properties of vitrimer matrices based on polydimethylsiloxane (PDMS), polystyrene (PS), and poly(methyl methacrylate) (PMMA). ${ }^{117,118}$ As a base case, we first consider PDMS with $N=N_{e}$ and $N_{x}=10$ uniformly spaced XLs. We set $E_{a}^{\mathrm{sm}}=28.1 \mathrm{~kJ} / \mathrm{mol}$, corresponding to the upper bound of measured activation energies of metathesis between small molecule dioxaborolanes. ${ }^{22}$ Since $G_{0}$ and $\tau_{b}$ are also functions of temperature, we select a reference temperature $T_{r}=413 \mathrm{~K}$, and express the modulus and time by normalizing with $G_{0}^{r}=G_{0}\left(T_{r}\right)=\rho R T_{r} / M_{0}$ and $\tau_{b}^{r}=\tau_{b}\left(T_{r}\right)$, respectively. To decouple the temperature dependence of the XL exchange kinetics and monomer friction, we define an Arrhenius shift factor $\left(e_{T}\right)$ that is analogous to the WLF shift factor $\left(a_{T}\right.$, eqn. 20),

$$
\log e_{T}=\frac{E_{a}^{\mathrm{sm}}}{R}\left(\frac{1}{T}-\frac{1}{T_{r}}\right)
$$

Figure 6a shows the acceleration in the PDMS vitrimer dynamics as temperature is increased. For context, as $T$ increases from $313-463 \mathrm{~K}, \delta$ falls about $30 \times$ from $\sim 6000$ to $\sim 180$ (figure 7), while $G_{0} \sim T$. Thus, the change in the modulus is much weaker; it increases only by $50 \%$, even 


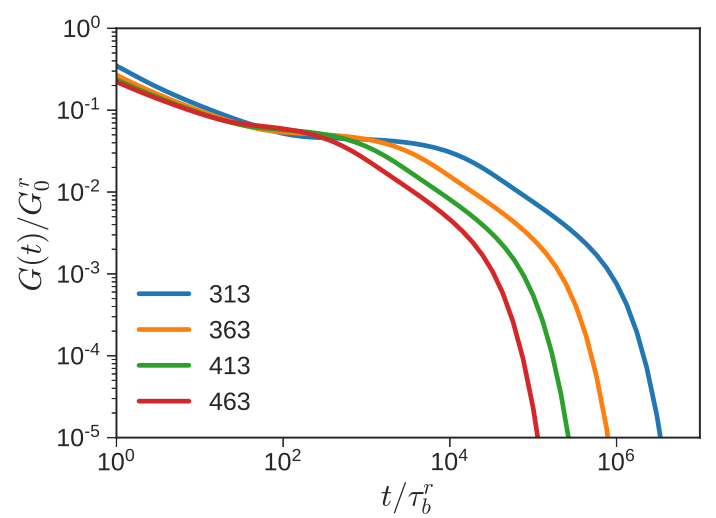

(a) unshifted

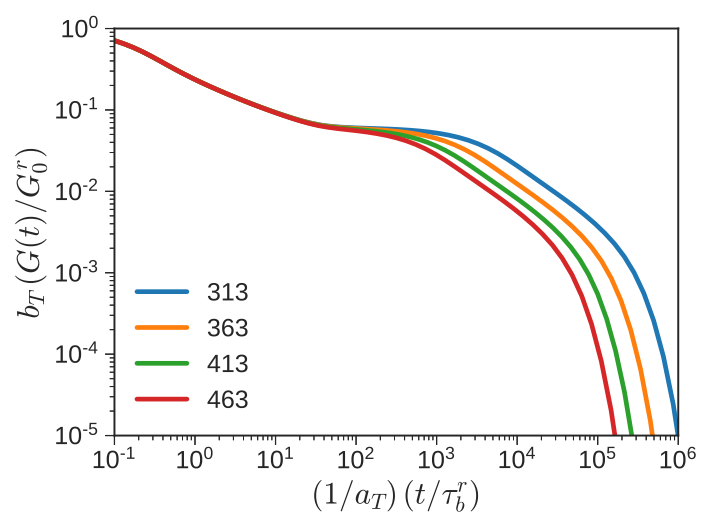

(c) WLF shift

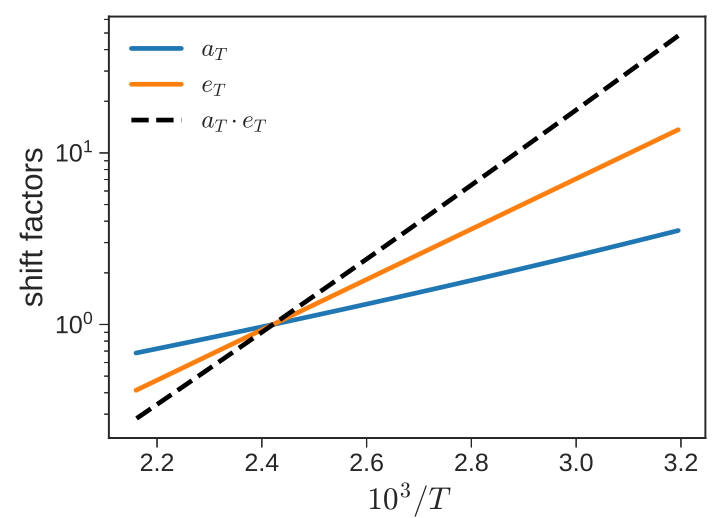

(b) shift factors

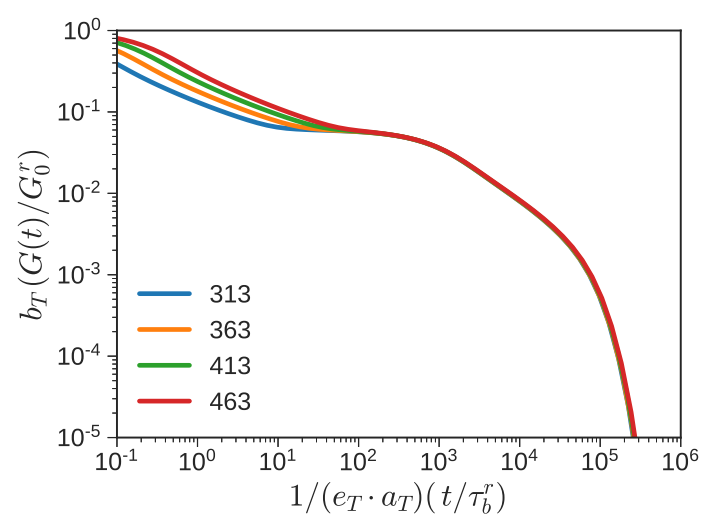

(d) WLF-Arrhenius shift

Figure 6: (a) Stress relaxation of PDMS with $N=N_{e}$ and $N_{x}=10$ XLs uniformly distributed along the chain as temperature is varied between 313-463 K. (b) The WLF and Arrhenius temperature shift factors. The response from (a) is shifted vertically, and horizontally using (c) WLF and (d) combined WLF-Arrhenius shift factors. 
over this artificially large window of temperature spanning $150 \mathrm{~K}$. On a log-log plot, this change is not clearly visible. Because the effect of temperature is manifested primarily through variation in $\delta$, figure 6 bears a qualitative resemblance to figure 4a. The plateau due to the XLs has a modulus that is insensitive to $\delta$, and is followed by Rouse-like relaxation.

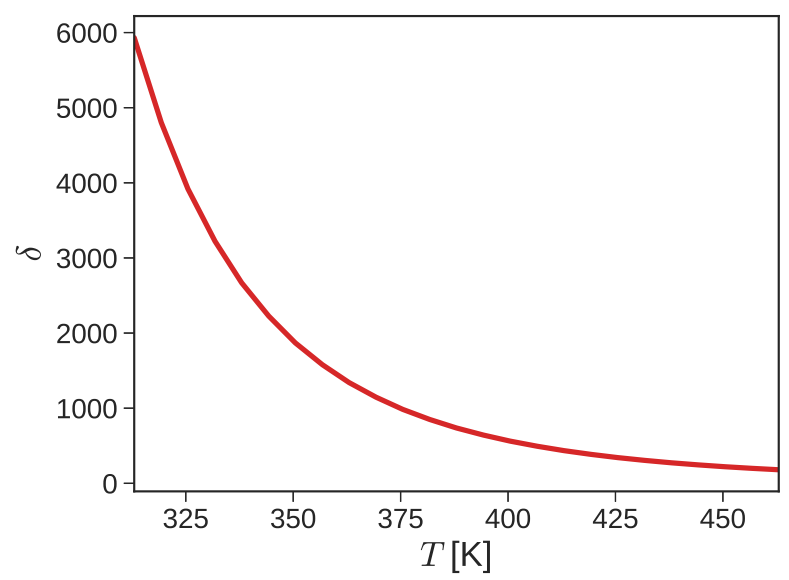

Figure 7: Variation of $\delta$ with temperature for PDMS samples in the temperature range 313 - 463 K. $\delta$ falls about $30 \mathrm{x}$ from $\sim 6000$ to $\sim 180$ over this temperature range.

Figure $6 \mathrm{~b}$ plots $a_{T}$ and $e_{T}$ for this system as a function of inverse temperature for $T=313-$ $463 \mathrm{~K}$. They intersect at $T_{r}=413 \mathrm{~K}$, where both $a_{T}$ and $e_{T}$ are equal to unity by definition. For PDMS, this temperature range is sufficiently removed from the glass transition temperature and $T_{\infty}=T_{0}-C_{2}=81 \mathrm{~K}$. Consequently, the WLF equation takes the approximate Arrhenius form given by eqn 21 , which is evident from the linear dependence of $\log a_{T}$ on $1 / T$ in the subfigure. For PDMS, the dependence of $a_{T}$ on temperature is weaker than the $e_{T}$ dependence, largely due to the small value for the coefficient $C_{1}$. As shown shortly, this is atypical; PS and PMMA, for example, have relatively high $T_{\infty}$ and $C_{1}$. If $\tau_{x}^{0}=2 \tau$, as assumed here, the overall dynamics are governed by $\tau_{x}$ through a product of $a_{T}$ (monomer friction) and $e_{T}$ (XL exchange). ${ }^{119}$ This is shown by the dashed black line in figure 6b. This dependence is stronger than either $a_{T}$ or $e_{T}$. Activation energies inferred from the slopes of $a_{T}, e_{T}$, and the product $a_{T} \cdot e_{T}$, are 11.5, 28.1, and $39.6 \mathrm{~kJ} / \mathrm{mol}$, respectively.

Figures 6c and 6d apply the horizontal and vertical shift factors to the PDMS vitrimer stress 
relaxation data. $b_{T}, a_{T}$, and $e_{T}$ are calculated using eqns 22,23 , and 24. Since the temperature dependence of monomer friction and XL dynamics are different, time-temperature superposition (TTS) cannot be performed using a single set of horizontal shift factors. When the curves are shifted using the WLF shift factor $a_{T}$ (figure 6c), only the short time dynamics superimpose ( $t \lesssim$ $\left.\tau_{x}^{0}\right)$. When the curves are shifted using the combined WLF-Arrhenius shift factor $e_{T}$ (figure 6d), the long time dynamics collapse $\left(t \gtrsim \tau_{x}^{0}\right)$. Concurrently, there is a dispersion at short times. This divergence in superposition suggests that the short and long time dynamics are governed by different processes. The short time dynamics are controlled by the mobility of the monomer, as specified by the WLF equation $\tau(T) / \tau\left(T_{r}\right)=a_{T}$, where $\tau\left(T_{r}\right)$ is the elementary Rouse timescale at the reference temperature. In this regime, the network is unaware of XL exchanges. The long time dynamics, however, are dictated by the combination of network strand relaxation and $\mathrm{XL}$ exchange. Mathematically, this implies that the longest relaxation time of the IHR model obeys the relationship $\tau_{1}(T) / \tau_{1}\left(T_{r}\right) \approx a_{T} \cdot e_{T}$.

Figure 8 depicts the variation in $\eta_{0}$ as a function of inverse temperature for PDMS, PS, and PMMA vitrimers that have $N=N_{e}$ and $N_{x}=10$ uniformly spaced XLs. $\eta_{0}$ is normalized by the product of $G_{0}$ and the monomer relaxation time at the reference temperature (which has units of viscosity). We neglect the change in polymer density, and assume $G_{0} \sim T$. In the figure, the temperature ranges used for PDMS, PS, and PMMA are $383-463 \mathrm{~K}, 463-600 \mathrm{~K}$, and $463-500$ $\mathrm{K}$, respectively. The degradation temperatures for PDMS and PS are around $600 \mathrm{~K}$, while it is only $500 \mathrm{~K}$ for PMMA. ${ }^{120,121}$ Thus, the upper range of temperatures explored for PS and PMMA are close to their respective degradation temperatures.

The four subplots span the spectrum of activation energies that have been observed for various vitrimer XL chemistries. $E_{a}^{\mathrm{sm}}=15$ and $30 \mathrm{~kJ} / \mathrm{mol}$ correspond to the lower and upper limits for small molecule dioxaborolane and imine metathesis. ${ }^{22} E_{a}^{\mathrm{sm}}=75 \mathrm{~kJ} / \mathrm{mol}$ and $100 \mathrm{~kJ} / \mathrm{mol}$ are consistent with transesterification. ${ }^{67}$ The range of the inverse temperature and normalized viscosity in the four subplots is kept identical to illustrate both the change in magnitude, and the slope as a function of $E_{a}^{\mathrm{sm}}$. By comparing each subplot, we observe that the viscosity changes by sev- 

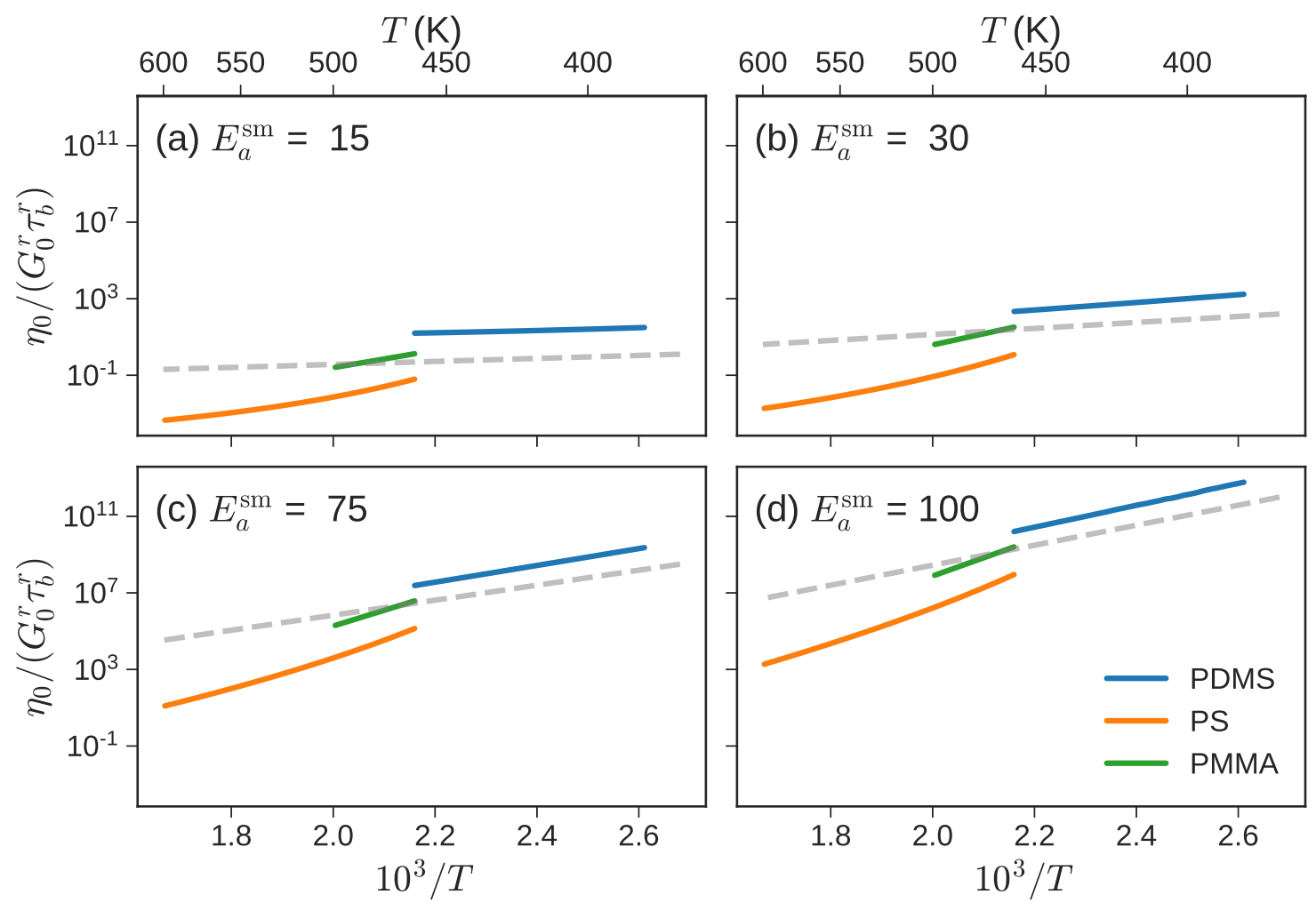

Figure 8: Solid lines depict viscosity as a function of inverse temperature of PDMS (blue), PS (orange) and PMMA (green) using $\tau_{x}^{0} \sim \tau$. The slope of the dashed lines is proportional to the small molecule exchange reaction activation energies $E_{a}^{\mathrm{sm}}=15,30,75$ and $100 \mathrm{~kJ} / \mathrm{mol}$, as indicated on the subplots. 
eral orders of magnitude as the activation energy increases. This is expected because viscosity is governed by the XL exchange reactions which slow down exponentially with increasing $E_{a}^{\mathrm{sm}}$.

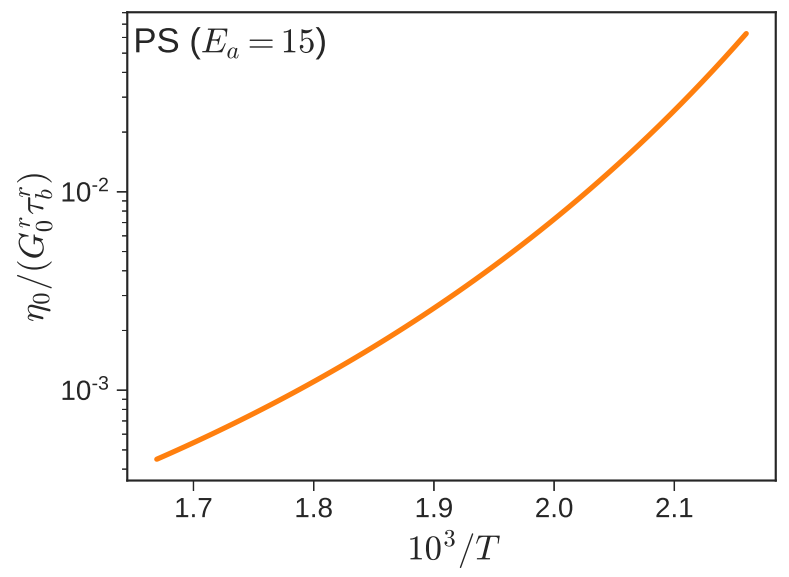

Figure 9: The temperature dependence for viscosity of PS, with $E_{a}^{\mathrm{sm}}=15 \mathrm{~kJ} / \mathrm{mol}$ from figure 7a is zoomed. The curvature at low temperatures (high $1 / T$ ) highlights the departure from Arrheniuslike behavior.

Figure 8 also highlights the different temperature responses for each vitrimer matrix. PDMS vitrimer exhibits the classical Arrhenius-type dependence $\log \eta_{0} \sim 1 / T$. This is related to figure 6b; at sufficiently high temperatures, WLF follows an apparent exponential form. The slope of $\eta_{0}$ versus $1 / T$ gives the activation energy from viscosity, $E_{a}^{\mathrm{rh}}$. In nearly all cases, $E_{a}^{\mathrm{rh}}>E_{a}^{\mathrm{sm}}$. This indicates that, in general, the activation energy inferred from rheology is larger than the activation energy obtained from small molecule studies. In contrast to PDMS, PS vitrimer exhibits curvature for $\eta_{0}$ versus $1 / T$, especially at low temperatures (high $1 / T$ ) (see figure 9). At low temperatures, WLF contributions are manifested by nonlinearity in the $\eta_{0}$ versus $1 / T$ plot. At sufficiently high temperatures, however, the non-Arrhenius behavior that arises from WLF modes can be visually masked over a limited temperature range. PMMA vitrimer demonstrates an apparent Arrheniustype dependence for $\eta_{0}$ due to the limited temperature range that is explored.

Figure 10 compares $E_{a}^{\mathrm{rh}}$ obtained from the figure 8 against the underlying $E_{a}^{\mathrm{sm}} \cdot E_{a}^{\mathrm{rh}}$ is extracted from the high temperature (low 1/T) part of the temperature window, where Arrhenius-type dependence is observed. The smallest discrepancy between $E_{a}^{\mathrm{rh}}$ and $E_{a}^{\mathrm{sm}}$ is observed for PDMS, 


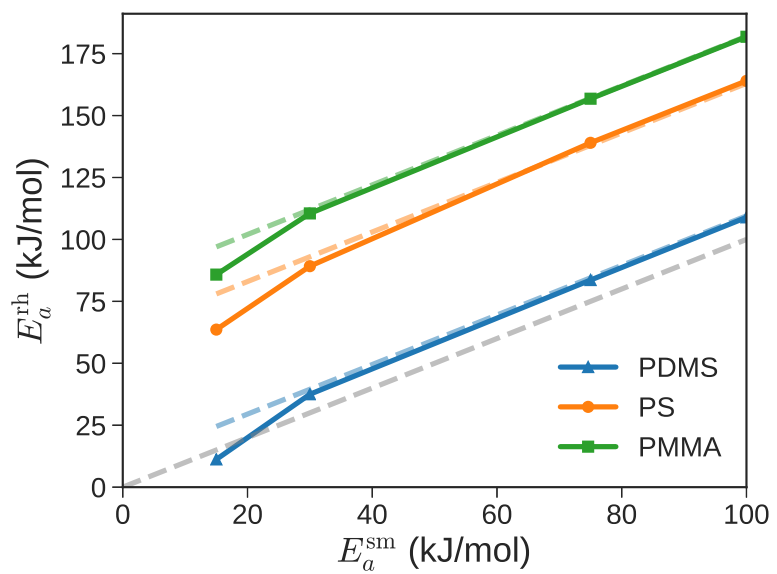

Figure 10: $E_{a}^{\mathrm{rh}}$ versus $E_{a}^{\mathrm{sm}}$ for the cases considered in figure 8 . The dashed gray line is the dependence expected for $E_{a}^{\mathrm{rh}}=E_{a}^{\mathrm{sm}}$. Dashed blue, orange, and green lines denote $E_{a}^{\mathrm{sm}}$ offset by 10,63 , and $82 \mathrm{~kJ} / \mathrm{mol}$, respectively. These offsets are approximately equal to $E_{\mathrm{WLF}}$ corresponding to the three polymers.

where the two energies deviate by about $15 \%$. This is consistent with the reasonably close correspondence between the slopes of the PDMS $\eta_{0}$ curves and the $E_{a}^{\mathrm{sm}}$ dashed lines in figure $8 . E_{a}^{\mathrm{rh}}$ increases from $\approx 12 \mathrm{~kJ} / \mathrm{mol}$ to $109 \mathrm{~kJ} / \mathrm{mol}$ as $E_{a}^{\mathrm{sm}}$ increases from 15 to $100 \mathrm{~kJ} / \mathrm{mol}$. The difference between $E_{a}^{\mathrm{rh}}$ and $E_{a}^{\mathrm{sm}}$ is larger for PS and PMMA. For activation energies of $30 \mathrm{~kJ} / \mathrm{mol}$ and above, the difference $E_{a}^{\mathrm{rh}}-E_{a}^{\mathrm{sm}} \approx E_{\mathrm{WLF}}$ is approximately constant and is equal to about $10 \mathrm{~kJ} / \mathrm{mol}$ for PDMS, $65 \mathrm{~kJ} / \mathrm{mol}$ for PS, and $80 \mathrm{~kJ} / \mathrm{mol}$ for PMMA. In this regime, a simple approximate relationship between the two activation energies exists, and is given by $E_{a}^{\mathrm{rh}} \approx E_{a}^{\mathrm{sm}}+E_{\mathrm{WLF}}$.

This is not surprising; it is a manifestation of the observation that terminal relaxation at different temperatures can be superposed by using the combined WLF-Arrhenius shift factors (see figure 6d, for example). Indeed, it can be traced back to the assumption of $\zeta_{x}=\zeta \delta$, or $\tau_{x}=\tau_{x}^{0} e^{E_{a}^{\mathrm{sm}} / R T}$. For temperatures sufficiently above $T_{g}, a_{T}$ approximately follows an Arrhenius relationship, as does the product $a_{T} \cdot e_{T}$ (see figure $6 \mathrm{~b}$ ). Note that deviations from $E_{a}^{\mathrm{rh}}=E_{a}^{\mathrm{sm}}+E_{\mathrm{WLF}}$ are expected when $T \sim T_{g}$, as $a_{T}$ does not follow an Arrhenius form at that temperature regime.

To summarize, the IHR model predicts that the vitrimer matrix and XL chemistry profoundly impact viscoelasticity. For all samples, the short time dynamics are controlled by monomer fric- 
tion, while the long time dynamics are governed by the combination of network strand relaxation and XL exchange. The viscosity of PDMS vitrimers, which have a fairly low $T_{g}$, demonstrates the expected Arrhenius temperature dependence. For PS vitrimers, which have high $T_{g}$, Arrhenius behavior is only displayed at very high temperatures. PMMA vitrimers exhibit Arrhenius behavior over the small temperature range that was explored. For temperatures sufficiently above $T_{g}$, the observed $E_{a}^{\mathrm{rh}}$ for all systems can be estimated a priori using the $E_{a}^{\mathrm{sm}}$ and WLF parameters, as $E_{a}^{\mathrm{rh}} \approx E_{a}^{\mathrm{sm}}+E_{\mathrm{WLF}}$, where $E_{\mathrm{WLF}}$ is given by eqn 21 . This explains the general observation $E_{a}^{\mathrm{rh}}>E_{a}^{\mathrm{sm}}$

\section{Discussion}

\subsection{Influence of Pre-exponential Factor on Temperature Dependence}

For the generalized sticky Rouse model approach presented in this manuscript, the drag on a sticky bead is proportional to $\tau_{x}=\tau_{x}^{0} e^{E_{a}^{\mathrm{sm}} / R T}$, where the pre-exponential factor $\tau_{x}^{0}=\sigma \tau$. We treated $\sigma$ as a constant, i.e., $\sigma=2$. While the functional form of $\tau_{x}^{0}$ is simple, it relies on two major assumptions: (i) $\sigma$ is independent of temperature and cross-link density, and (ii) the monomer relaxation time $\tau$ follows a WLF relationship with temperature.

The assumption of independence of $\sigma$ with temperature is consistent with established theoretical models of XL dynamics, ${ }^{115}$ but it neglects the elaborate choreography involved in bringing two XLs together to facilitate an exchange reaction. Associative cross-linking within a vitrimer is not a mean-field process; the density, spatial distribution and orientation of the cross-links most likely play an important role. As proposed by de Gennes, reactive groups attached to flexible polymer chains explore their surroundings via sub-diffusive and compact random walks. ${ }^{96}$ Based on this idea, the tethering of the vitrimer XLs to network strands reduces their mobility and probability of encountering another XL, reactive group, or catalyst. ${ }^{115,122,123}$ XLs may even reassociate with old partners in ways that do not relieve stress along the backbone. ${ }^{84}$ These impediments significantly delay terminal relaxation by orders of magnitude. ${ }^{124}$ Although the influence of vitrimer structure 
on XL mobility is outside the scope of this study, it can potentially be evaluated through the IHR or SSR models by treating $\sigma$ as a fitting parameter rather than a constant. Precise calculation of $\sigma$ will permit accurate estimation of processing parameters, e.g., $\eta_{0}$ and $T_{v}$. However, we must emphasize that like the standard Rouse model, the IHR is fundamentally a single chain model. It cannot directly account for nontrivial multi-chain interactions.

At the moment, direct comparison of the IHR model to vitrimer rheology data in the literature is limited due to both (i) the current state of experimental data on unentangled vitrimers and (ii) the model itself (in particular, $\sigma$ ). Experimental studies on vitrimer systems in which $E_{a}^{\text {rh }}$ and $E_{a}^{\mathrm{sm}}$ are separately measured are complied in Table S2 of Supporting Information. In all of these studies, the vitrimer chains are nominally entangled, which makes them unsuitable to test the IHR. The second reason that impedes direct comparison with experiments is the uncertainty of $\tau_{x}^{0}$. By assuming $\tau_{x}^{0}=\sigma \tau$ with $\sigma=2$, the dependence of the prefactor $\tau_{x}^{0}$ on cross-link density, spatial distribution of cross-links, etc., is ignored. As long as $\sigma$ is independent of temperature, claims about Arrhenius behavior and the temperature dependence of the terminal dynamics ought to remain valid. However, the predicted values of $\tau_{\eta}$ and $\tau_{1}$ are likely underestimations. For these reasons, we believe that more rigorous rheology data sets on model unentangled vitrimer systems are needed to fairly test the IHR theory and learn more about the form and magnitude of $\sigma$.

The WLF assumption, standard for polymeric systems, establishes the combined temperature dependence of $\tau$ and the exponential factor. Consequently, $E_{a}^{\mathrm{rh}}$ is predicted to be greater than $E_{a}^{\mathrm{sm}}$ for PDMS, PMMA, and PS vitrimers. The difference between two activation energies is approximately equal to $E_{\mathrm{WLF}}$, especially for $T \gg T_{g}$. For PDMS, $E_{\mathrm{WLF}} \approx 10 \mathrm{~kJ} / \mathrm{mol}$, the difference between the two activation energies is within experimental uncertainty. For PMMA and PS, $E_{\mathrm{WLF}}$ is approximately equal to $65 \mathrm{~kJ} / \mathrm{mol}$ and $80 \mathrm{~kJ} / \mathrm{mol}$, respectively, making it greater than $E_{a}^{\mathrm{sm}}$ in some cases. This relationship among $E_{a}^{\mathrm{rh}}, E_{a}^{\mathrm{sm}}$, and $E_{\mathrm{WLF}}$ provides a simple and convenient method for predicting viscosity. However, the mechanism of the XL exchange within the vitrimer matrix will impact the relationship between $E_{a}^{\mathrm{rh}}$ and $E_{a}^{\mathrm{sm}}$. If another process that has a weaker temperature dependence is rate-controlling, e.g., proper alignment of reacting elements, then $\tau_{x}^{0} \sim \tau$ may no 
longer be valid. In such a situation, $\sigma$ would dominate $\tau_{x}^{0}$. Small molecule diffusion, which typically has an Arrhenius temperature dependence, ${ }^{56}$ may also mediate XL exchange in externally catalyzed vitrimers. If catalyst transport is the rate limiting step for relaxation, then $\tau_{x}^{0}$ inherits the Arrhenius relationship. A plot of $\log \eta_{0}$ versus $1 / T$ would be linear, but the slope of the line would be a function of the activation energy for catalyst diffusion.

In some of these situations it is quite possible that $\sigma$ is temperature-dependent. If the $\tau_{x}=$ $\tau_{x}^{0} e^{E_{a}^{\mathrm{sm}} / R T}$ form is still valid then we obtain,

$$
\begin{aligned}
\ln \tau_{x} & =\ln \tau+\ln \sigma+\frac{E_{a}^{\mathrm{sm}}}{R T} \\
\frac{\partial}{\partial(1 / T)} \ln \tau_{x} & =\frac{\partial}{\partial(1 / T)} \ln \tau+\frac{\partial}{\partial(1 / T)} \ln \sigma+\frac{\partial}{\partial(1 / T)} \frac{E_{a}^{\mathrm{sm}}}{R T} \\
E_{a}^{\mathrm{rh}} & =E_{\mathrm{WLF}}+E_{a}^{\sigma}+E_{a}^{\mathrm{sm}}
\end{aligned}
$$

where $E_{a}^{\sigma}$ is the activation energy corresponding to $\sigma(T)$. In such situations, $E_{a}^{\sigma}$ may be an important parameter that encodes interesting physics underlying the XL exchange.

\subsection{Recommended Practices to Extract Accurate $\mathrm{E}_{\mathrm{a}}^{\mathrm{rh}}$}

Conceptually, vitrimers are viscoelastic liquids. At sufficiently high temperatures and long timescales they reach terminal relaxation, and their relaxation corresponds to a unique zero-shear viscosity $\eta_{0}$. As mentioned previously, $\tau_{\eta}$ and $\eta_{0}$ depend on the slowest relaxation mode, $\tau_{1}$. Thus, timespans of $t>\tau_{1}$ need to be evaluated to accurately probe these parameters. Otherwise, the estimated $\tau_{\eta}$ and $\eta_{0}$ are not unique, and are influenced by the method of both measurement and analysis.

Figure 11 depicts simulated vitrimer linear viscoelasticity data for various types of rheology experiments: stress relaxation, creep, and small amplitude oscillatory shear. To find $\eta_{0}$, one must first check if the sample has indeed completely relaxed by looking for a characteristic rheological signature. Depending on the experiment used, this is manifested in different ways. For stress relaxation, $\log G(t) \sim t$; for creep compliance, $J(t) \sim t$; for small amplitude oscillatory shear (SAOS), 
$G^{\prime}(\omega) \sim \omega^{2}$ and $G^{\prime \prime}(\omega) \sim \omega$. Caution must be exercised in interpreting $\eta_{0}$ obtained when these signatures of terminal relaxation cannot be ascertained. While stress relaxation/SAOS measurements over long-time/low-frequency scales are limited by the torque resolution of the rheometer, creep does not suffer from this issue, making it especially well-suited for characterizing terminal properties.
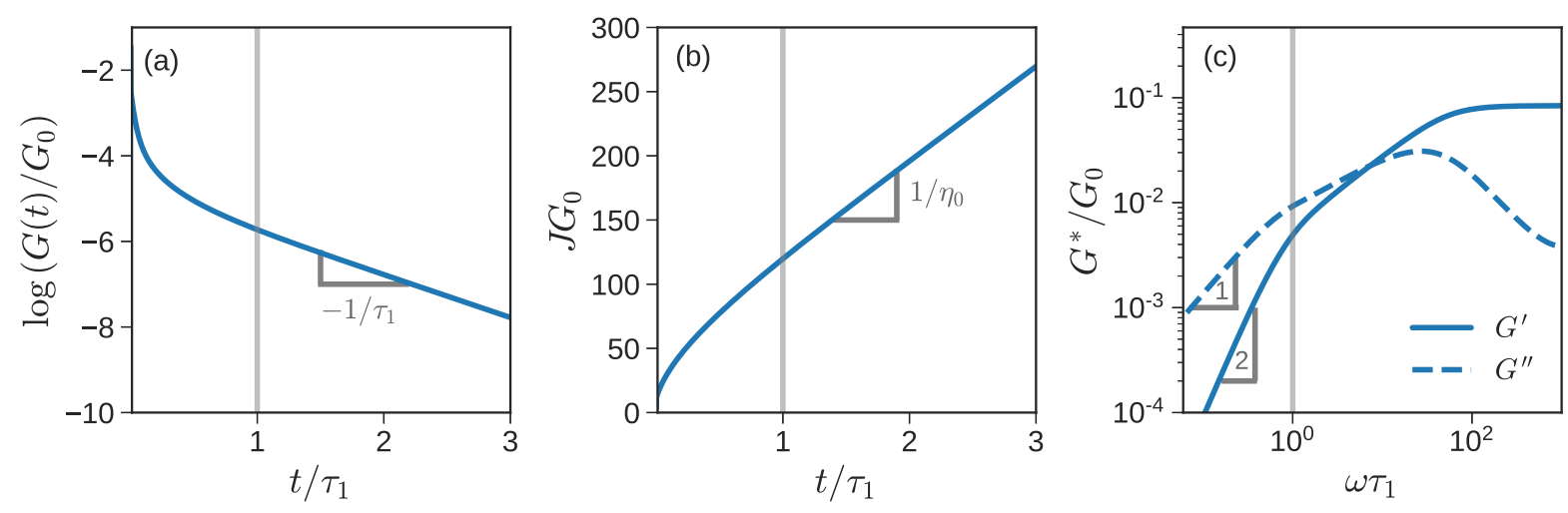

Figure 11: Normalized (a) $G(t)$, (b) $J(t)$, and (c) $G^{*}(\omega)$ are replotted for the $\delta=10^{4}$ sample considered in figure 4(a) to highlight signs of terminal relaxation. The horizontal time or frequency axis is normalized by the longest relaxation time $\tau_{1}$ (marked by vertical gray lines) instead of $\tau_{b}$ to emphasize the behavior in the terminal regime. For $t>\tau_{1}$ and $\omega<1 / \tau_{1}$, signatures of terminal relaxation are observed, viz. $\log G(t) \sim-t / \tau_{1}, J(t) \sim t / \eta_{0}$, and $G^{\prime} \sim \omega^{2}$ and $G^{\prime \prime} \sim \omega$.

Figure 11a depicts the $G(t), J(t)$, and $G^{*}(\omega)$ for the $\delta=10^{4}$ sample previously considered in figure 4a, where $G(t)$ was shown on a $\log -\log$ plot. To convert $G(t)$ to $J(t)$ in figure $11 \mathrm{~b}$ we used the spectrum of relaxation times $\left\{\tau_{i}\right\}$ obtained from the IHR model, and performed interconversion to creep compliance using the Prony series method. ${ }^{125,126}$ As evident from figure 11, we need to probe times of the order of $2-5 \tau_{1}$ to ascertain characteristic signatures of terminal relaxation associated with these measurements.

Although accurate evaluation of $E_{a}^{\text {rh }}$ requires the sample to reach terminal relaxation, rheological measurements on vitrimer systems have been typically run for inadequate timespans. Failure to reach $t \gtrsim \tau_{1}$ can create systematic errors in the estimation of $E_{a}^{\text {rh }}$. To emphasize this point with a concrete example, we reconsider the PDMS sample used in figure 6a. Recall that for this sample, $N=N_{e}, N_{x}=10$ (uniformly dispersed XLs), and $E_{a}^{\mathrm{sm}}=28.1 \mathrm{~kJ} / \mathrm{mol}$. 

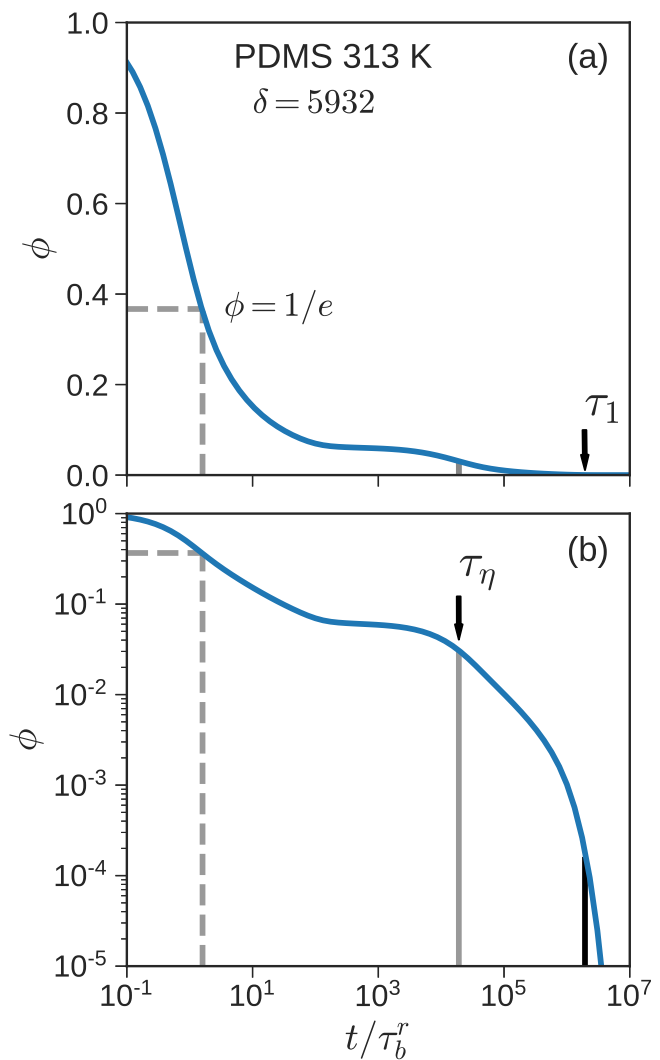

Figure 12: Normalized stress relaxation modulus for PDMS at $313 \mathrm{~K}$ plotted on (a) linear and (b) logarithmic scales. The location where $\phi(t)$ falls to $1 / e$ of its initial value is indicated by dashed lines. Locations of the longest and average relaxation times $\tau_{1}$ and $\tau_{\eta}$ are indicated by arrows in (a) and (b), respectively. 
Figure 12 plots the normalized stress relaxation function $\phi(t)$ at a particular temperature, viz. the $T=313 \mathrm{~K}$ sample, with linear and logarithmic vertical axes. The horizontal axis is common and logarithmic. A popular, but ill-advised, procedure for determining the characteristic relaxation time $\tau^{*}$ from stress relaxation experiments is to define it as the time point where $\phi(t)$ falls to $1 / e$ of its original value (marked by dashed gray lines in the figure). Fundamentally, this threshold is appropriate if relaxation is governed by a single Maxwell mode (in which case, $\tau^{*}=\tau_{1}=\tau_{\eta}$ ). For polymeric systems with a wide spectrum of relaxation times, however, this is inaccurate. In particular, for vitrimer systems this method runs the risk of probing timescales much shorter than $\tau_{x}^{0}$, and underestimating $\eta_{0}$ by several orders of magnitude. As shown in the figure, the difference between these characteristic timescales defined as either $\phi\left(\tau^{*}\right)=1 / e$ or as the longest relaxation time $\tau^{*}=\tau_{1}$ (marked in black), is over five decades. More problematically, using the $1 / e$ threshold complicates the analysis of the temperature dependence of $\tau^{*}$. As observed in figure 6, TTS cannot be obtained using a single set of parameters. Any estimates of $\tau^{*}$ obtained by probing $t<\tau_{x}^{0}$ only reveal the influence of temperature on the elementary Rouse timescale $\tau$ (and perhaps $\tau_{x}^{0}$ ), but not $\tau_{x}$ or $\tau_{1}$.

Figure 13 shows how the method used to specify the characteristic relaxation time $\tau^{*}$ impacts the estimated activation energy $E_{a}^{\mathrm{rh}}$. Since $E_{a}^{\mathrm{rh}}$ is obtained from the slope of $\log \tau^{*}$ versus $1 / T$, the (logarithmic) vertical axis is normalized by $\tau^{*}$ at the reference temperature $T_{r}=413 \mathrm{~K}\left(\tau_{r}^{*}\right)$, which causes different curves to pass through a common point. When $\tau^{*}$ is defined through $\phi\left(\tau^{*}\right)=1 / e$ (blue), the activation energy estimated from the slope is comparable with $E_{\mathrm{WLF}} \approx 11 \mathrm{~kJ} / \mathrm{mol}$ which is shown by the dashed line. This is not surprising, since $1 / e \approx 0.37$ is greater than the $G_{x} / G(0) \approx 0.1$ plateau in fig 12 which is associated with the XLs. Thus, $\tau^{*}$ determined using this criterion effectively probes the temperature dependence of short time dynamics, i.e., chain friction. On the other hand, the $E_{a}^{\mathrm{rh}}$ estimated when $\tau^{*}$ is defined as either the average $\left(\tau_{\eta}\right)$ or longest relaxation time $\left(\tau_{1}\right)$ are identical. It corresponds quite well with the activation energy estimated using $E_{a}^{\mathrm{rh}}=E_{a}^{\mathrm{sm}}+E_{\mathrm{WLF}} \approx 40 \mathrm{~kJ} / \mathrm{mol}$, shown by the dotted line.

This analysis suggests a useful rule of thumb when the rubbery plateau $G_{x}$ corresponding to 
the XLs is visible on a log-log plot. Any timescale that probes $G(t)<G_{x}$ such as $\tau_{\eta}$ or $\tau_{1}$ in figure $12 \mathrm{~b}$ is a good proxy for the characteristic relaxation time $\tau^{*}$ for extracting $E_{a}^{\mathrm{rh}}$. Interestingly, defining $\tau^{*}$ implicitly via $G\left(\tau^{*}\right) / G_{x}=1 / e$ instead of $G\left(\tau^{*}\right) / G_{0}=1 / e$ avoids the problem noted in figure 13. However, it should be noted that experimentally it may be difficult to determine $G_{x}$ and probe timescales long enough to observe terminal relaxation. This is especially true when $\delta$ is large, and $N_{x} / N$ is small. In entangled vitrimers, additional complexity arises because $G_{x}$ combines with the plateau modulus $G_{N}^{0}$ that arises from entanglements. The example considered in figures 12 and 13 presents a case where the contributions of monomer mobility $(\tau)$ and XL lifetime $\left(\tau_{x}\right)$ are well-separated. In some systems, however, these relaxation modes may overlap. Resolving these modes experimentally - i.e., by exploring a large temperature/time scale window - may be hampered due to the thermal sensitivity of the sample. In such situations, simulations of the IHR model may be used in conjunction with experiment to tease apart contributions from the different relaxation mechanisms.

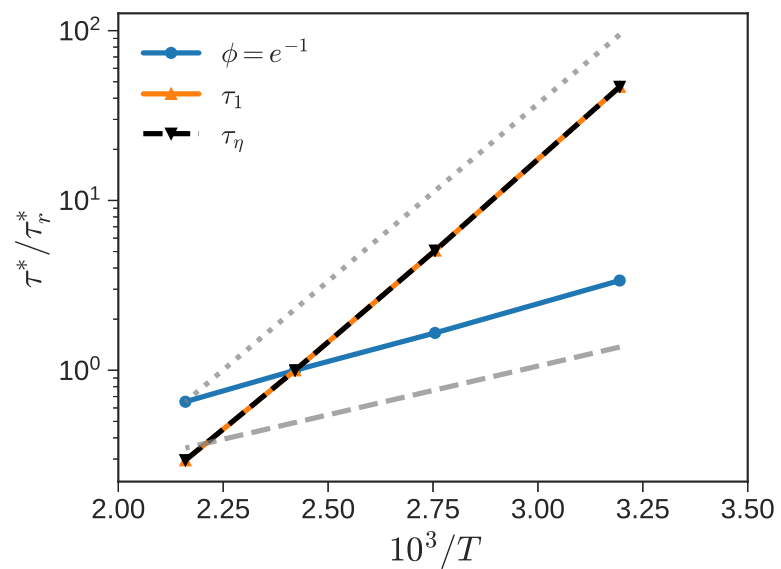

Figure 13: Arrhenius plot of the characteristic relaxation time $\tau^{*}$, determined using three different methods, versus inverse temperature for the PDMS samples shown in figure 6a. $\tau^{*}$ is normalized by the characteristic relaxation time at $T_{r}=413 \mathrm{~K}\left(\tau_{r}^{*}\right)$ to facilitate comparison of slopes. The overlapping orange and dashed black lines correspond to $\tau_{1}$ and $\tau_{\eta}$ respectively. Blue circles denote $\tau^{*}$ obtained using $\phi\left(\tau^{*}\right)=1 / e$. The slopes of the dashed and dotted gray lines correspond to $E_{\mathrm{WLF}} \approx 11 \mathrm{~kJ} / \mathrm{mol}$, and $E_{a}^{\mathrm{rh}}=E_{a}^{\mathrm{sm}}+E_{\mathrm{WLF}} \approx 40 \mathrm{~kJ} / \mathrm{mol}$, respectively. 


\subsection{Utility and Limitations of IHR and SSR Models for Vitrimer Systems}

The IHR and SSR models are powerful tools for forecasting and analyzing vitrimer viscoelasticity. They capture many of the rheological signatures that have been observed experimentally (e.g., Arrhenius temperature dependence, secondary peaks and plateaus in small amplitude oscillatory shear, strong dependence on the matrix and XL chemistry). Based only on knowledge of the vitrimer composition and XL exchange kinetics, they predict the linear viscoelastic response across the entire time/frequency domain for multiple types of measurement techniques. To achieve a specific rheological profile, the optimal pairing of XL structure and backbone can be identified a priori. Moreover, these models offer insight into the network topology and dynamics. The presence of defects in the vitrimer network may be probed by comparing the measured plateau modulus $G_{x}$ to the anticipated value from rubber elasticity theory. ${ }^{127-130} \sigma$ potentially serves as a proxy for XL mobility, as mentioned earlier. Thus, the IHR and SSR can serve as platforms for connecting macroscopic flow behavior (as determined by rheology) to microscopic dynamics (as observed by dielectric relaxation spectroscopy or other techniques).

While these models can be used to advance understanding of structure-property relationships, they do have limitations. Generalized Rouse models like the IHR or SSR only apply to homogeneous vitrimers composed of unentangled linear chains; the XLs solely interact with the backbone monomers via the propagation of frictional drag. In real vitrimers, however, branches and entanglements enhance the elasticity and delay relaxation. ${ }^{43-46}$ Strong thermodynamic interactions between the XLs and backbone induce concentration fluctuations and macro/microphase separation. ${ }^{12,13,47-49}$ Slip link models have the capability to resolve multi-chain interactions in entangled polymer melts, and early work shows much promise. ${ }^{73}$ They are sufficiently coarse-grained to access long relaxation times observed at low temperatures, while retaining the ability to model the complex interchain dynamics. ${ }^{131-133}$ Furthermore, as seen from the comparison of the SSR and IHR models, if the timescales associated with chain relaxation and kinetics of exchange reactions are well-separated, we can envision a hierarchy of additive models, ranging from molecular dynamics that capture fine details of chain motion to slip link models that resolve terminal relaxation. 
In this work, we identify the conditions under which vitrimer viscosity follows an Arrhenius temperature dependence. Yet, vitrimers are not unique in this regard. Many types of polymeric systems have Arrhenius rheology. ${ }^{134-137}$ In particular, polymer networks with dissociative covalent XLs express this relationship because their network connectivity hinges on the equilibrium balance between the bonded/debonded states of the XLs. ${ }^{3}$ This mechanism is identical to that of the dynamic polymer networks outlined in the original sticky Rouse and sticky reptation papers. ${ }^{82-85}$ As such, the IHR and SSR models presented here can be adapted for systems with dissociative covalent XLs, but the XL density $N_{x}$ must be modified so that it is a function of temperature.

The similarity in rheological behavior, however, does not mean vitrimers and dissociative covalent polymer networks are the same. Vitrimer XLs maintain network connectivity at all temperatures, rendering them insoluble in good solvents. In contrast, dissociative covalent polymer networks will dissolve. Vitrimers are unique among polymers because of their combination of insolubility and processability. Both properties are necessary criteria for determining if a material is a vitrimer. It is not sufficient to only characterize their rheology.

\section{Conclusions}

The IHR model for unentangled, fully developed vitrimer networks describes the interplay between chain friction and XL exchange. When the lifetime of a XL $\left(\tau_{x}\right)$ is modeled as a product of WLF and Arrhenius contributions, the effective activation energy determined from rheology, $E_{a}^{\text {rh }}$, is typically larger than the activation energy for the exchange reaction between small molecule analogues, $E_{a}^{\mathrm{sm}}$. The difference between $E_{a}^{\mathrm{rh}}$ and $E_{a}^{\mathrm{sm}}$ depends strongly on the chemistry of the polymer matrix and the temperature range investigated, even when the XL chemistry is the same. At sufficiently high temperatures, $E_{a}^{\mathrm{rh}} \approx E_{a}^{\mathrm{sm}}+E_{\mathrm{WLF}}$. For PDMS vitrimer, $E_{\mathrm{WLF}}$ is relatively small, and $E_{a}^{\mathrm{rh}} \approx E_{a}^{\mathrm{sm}}$. On the other hand, for PMMA vitrimers, $E_{\mathrm{WLF}} \approx 80 \mathrm{~kJ} / \mathrm{mol}$ is large, resulting in substantial differences between $E_{a}^{\mathrm{rh}}$ and $E_{a}^{\mathrm{sm}}$. Similar trends are observed for PS vitrimers; however, at the lower end of the temperatures probed, non-Arrhenius behavior is observed. Estimation 
of $E_{a}^{\text {rh }}$ from an Arrhenius plot depends on the determination of $\eta_{0}$ or $\tau^{*}$. Systematic errors that arise from some common but ill-advised methods of data analysis are illustrated using the IHR model.

As a consequence of WLF and Arrhenius contributions, time-temperature superposition cannot be achieved over the entire relaxation spectrum. Use of WLF shift factors allows superposition of short time dynamics, which corresponds to relaxation of individual monomers. In contrast, a combination of WLF and Arrhenius shift factors are required to obtain superposition of long time relaxations due to the interplay between backbone relaxations and XL exchanges. This is similar to observations in other dynamic polymer network systems.

A comparison of the SSR and IHR model indicates that the SSR is a good approximation when three conditions are met: (i) $N_{x} \gg 1$, (ii) $\tau_{x} N_{x}^{2} \gg \tau N^{2}$, so that the sticky modes are well-separated from the regular Rouse modes, and (iii) the distribution of XLs is random or uniform. However, when any of these conditions is violated, use of the IHR model is advised. The rheology of a sticky Rouse model with random and uniformly distributed sticky beads are expected to be within experimental error limits, especially as $N_{x}$ increases. However, gradient and block distributions are quite different, and typically relax much faster than random and uniform distributions.

Vitrimers are a unique class of polymers, and there is still much left to be learned about their flow behavior. The first decade of vitrimer research has enabled the preparation of well-defined model materials in scalable amounts. Consequently, research efforts are now shifting towards establishing critical structure-viscoelasticity relationships. Inspired by previous achievements in the field of dynamic polymer networks, we believe that the use of more sophisticated network models offers one pathway towards success in this goal. Deep understanding of vitrimer flow and processability can only be achieved through a union of rigorous synthesis, experimental characterization, and modeling. 


\section{Supporting Information}

(i) Mathematical Notation for Different Timescales and (ii) Experimental Data on Vitrimer Systems.

\section{Acknowledgments}

This work is based in part upon work supported by the National Science Foundation under grant no. NSF DMR-1727870 (SS). We also acknowledge Florida State University and the FAMU-FSU College of Engineering for support.

\section{References}

(1) Montarnal, D.; Capelot, M.; Tournilhac, F.; Leibler, L. Silica-like malleable materials from permanent organic networks. Science 2011, 334, 965-968, DOI: $10.1126 /$ science.1212648.

(2) Scheutz, G. M.; Lessard, J. J.; Sims, M. B.; Sumerlin, B. S. Adaptable crosslinks in polymeric materials: Resolving the intersection of thermoplastics and thermosets. J. Am. Chem. Soc. 2019, 141, 16181-16196, DOI: 10 . 1021 / jacs. 9b07922.

(3) Winne, J. M.; Leibler, L.; Du Prez, F. E. Dynamic covalent chemistry in polymer networks: a mechanistic perspective. Polym. Chem. 2019, 10, 6091-6108, DOI: 10.1039/C9PY01260E.

(4) Guerre, M.; Taplan, C.; Winne, J. M.; Du Prez, F. E. Vitrimers: Directing chemical reactivity to control material properties. Chem. Sci. 2020, 11, 4855-4870, DOI: $10.1039 / \mathrm{d} 0 \mathrm{sc} 01069 \mathrm{c}$.

(5) Van Zee, N. J.; Nicolaÿ, R. Vitrimers: Permanently crosslinked polymers 
with dynamic network topology. Prog. Polym. Sci. 2020, 104, 101233, DOI: $10.1016 / j . p r o g p o l y m s c i .2020 .101233$.

(6) Parada, G. A.; Zhao, X. Ideal reversible polymer networks. Soft Matter 2018, 14, 51865196, DOI: $10.1039 / \mathrm{C} 8 \mathrm{SM} 00646 \mathrm{~F}$.

(7) Zhang, Y.; Broekhuis, A. A.; Picchioni, F. Thermally self-healing polymeric materials: the next step to recycling thermoset polymers? Macromolecules 2009, 42, 1906-1912, DOI: $10.1021 / \mathrm{ma} 8027672$.

(8) Scott, T. F.; Schneider, A. D.; Cook, W. D.; Bowman, C. N. Photoinduced plasticity in crosslinked polymers. Science 2005, 308, 1615-1617, DOI: 10 .1126/science. 1110505.

(9) Röttger, M.; Domenech, T.; van der Weegen, R.; Breuillac, A.; Nicolaÿ, R.; Leibler, L. Highperformance vitrimers from commodity thermoplastics through dioxaborolane metathesis. Science 2017, 356, 62-65, DOI: 10 .1126/science . aah5281.

(10) Nishimura, Y.; Chung, J.; Muradyan, H.; Guan, Z. Silyl ether as a robust and thermally stable dynamic covalent motif for malleable polymer design. J. Am. Chem. Soc. 2017, 139, 14881-14884, DOI: $10.1021 /$ jacs. 7b08826.

(11) Demongeot, A.; Groote, R.; Goossens, H.; Hoeks, T.; Tournilhac, F.; Leibler, L. Cross-linking of poly(butylene terephthalate) by reactive extrusion using Zn(II) epoxy-vitrimer chemistry. Macromolecules 2017, 50, 6117-6127, DOI: $10.1021 /$ acs.macromol.7b01141.

(12) Ricarte, R. G.; Tournilhac, F.; Leibler, L. Phase separation and self-assembly in vitrimers: Hierarchical morphology of molten and semicrystalline polyethylene/dioxaborolane maleimide systems. Macromolecules 2019, 52, 432-443, DOI: $10.1021 /$ acs .macromol.8b02144. 
(13) Ricarte, R. G.; Tournilhac, F.; Clotre, M.; Leibler, L. Linear viscoelasticity and flow of selfassembled vitrimers: the case of a polyethylene/dioxaborolane system. Macromolecules 2020, 53, 1852-1866, DOI: 10.1021 /acs.macromol.9b0 2415.

(14) He, C.; Shi, S.; Wu, X.; Russell, T. P.; Wang, D. Atomic force microscopy nanomechanical mapping visualizes interfacial broadening between networks due to chemical exchange reactions. J. Am. Chem. Soc. 2018, 140, 6793-6796, DOI: 10.1021 / jacs. 8b0 0371.

(15) Lessard, J. J.; Garcia, L. F.; Easterling, C. P.; Sims, M. B.; Bentz, K. C.; Arencibia, S.; Savin, D. A.; Sumerlin, B. S. Catalyst-free vitrimers from vinyl polymers. Macromolecules 2019, 52, 2105-2111, DOI: 10.1021 /acs.macromol. 8b 02477.

(16) Lessard, J. J.; Scheutz, G. M.; Hughes, R. W.; Sumerlin, B. S. Polystyrene-based vitrimers: inexpensive and recyclable thermosets. ACS Appl. Polym. Mater. 2020, 2, 3044-3048, DOI: 10.1021 /acsapm.0c00523.

(17) Taplan, C.; Guerre, M.; Winne, J. M.; Du Prez, F. E. Fast processing of highly crosslinked, low-viscosity vitrimers. Mater. Horiz. 2020, 7, 104-110, DOI: 10.1039 / c9mh 01062 a.

(18) Capelot, M.; Unterlass, M. M.; Tournilhac, F.; Leibler, L. Catalytic control of the vitrimer glass transition. ACS Macro Lett. 2012, 1, 789-792, DOI: 10.1021 /mz 300239 f.

(19) Capelot, M.; Montarnal, D.; Tournilhac, F.; Leibler, L. Metal-catalyzed transesterification for healing and assembling of thermosets. J. Am. Chem. Soc. 2012, 134, 7664-7667, DOI: $10.1021 / \mathrm{ja} 302894 \mathrm{k}$.

(20) Capelot, M. Chimie de polycondensation, polymères supramoléculaires et vitrimères. Ph.D. thesis, Université Pierre et Marie Curie - Paris VI, 2013.

(21) Denissen, W.; Rivero, G.; Nicolaÿ, R.; Leibler, L.; Winne, J. M.; Du Prez, F. E. Vinylogous urethane vitrimers. Adv. Funct. Mater. 2015, 25, 2451-2457, DOI: 10.1002 /adfm. 201404553. 
(22) Röttger, M. Associative exchange reactions of boron or nitrogen containing bonds and design of vitrimers. Theses, Université Pierre et Marie Curie - Paris VI, 2016.

(23) Hendriks, B.; Waelkens, J.; Winne, J. M.; Du Prez, F. E. Poly(thioether) vitrimers via transalkylation of trialkylsulfonium salts. ACS Macro Lett. 2017, 930-934, DOI: 10.1021 /acsmacrolett. 7b00494.

(24) Ishibashi, J. S. A.; Kalow, J. A. Vitrimeric silicone elastomers enabled by dynamic meldrum's acid-derived cross-links. ACS Macro Letters 2018, 7, 482-486, DOI: 10.1021 /acsmacrolett.8b00166.

(25) He, C.; Shi, S.; Wang, D.; Helms, B. A.; Russell, T. P. Poly(oxime-ester) vitrimers with catalyst-free bond exchange. J. Am. Chem. Soc. 2019, 141, 13753-13757, DOI: $10.1021 /$ jacs.9b06668.

(26) El-Zaatari, B. M.; Ishibashi, J. S. A.; Kalow, J. A. Cross-linker control of vitrimer flow. Polym. Chem. 2020, DOI: $10.1039 /$ d0py00233j.

(27) Brutman, J. P.; Delgado, P. A.; Hillmyer, M. A. Polylactide vitrimers. ACS Macro Lett. 2014, 3, 607-610, DOI: $10.1021 / \mathrm{mz} 500269 \mathrm{w}$.

(28) Altuna, F. I.; Hoppe, C. E.; Williams, R. J. Shape memory epoxy vitrimers based on DGEBA crosslinked with dicarboxylic acids and their blends with citric acid. $R S C A d v . \mathbf{2 0 1 6}, 6$, 88647-88655, DOI: $10.1039 /$ c6ra18010h.

(29) Stukenbroeker, T.; Wang, W.; Winne, J. M.; Du Prez, F. E.; Nicola, R.; Leibler, L. Polydimethylsiloxane quenchable vitrimers. Polym. Chem. 2017, 8, 6590-6593, DOI: $10.1039 /$ C7PY01488K.

(30) Altuna, F. I.; Hoppe, C. E.; Williams, R. J. J. Epoxy vitrimers: the effect of transesterification reactions on the network structure. Polymers 2018, 10, 43, DOI: $10.3390 /$ polym10010043. 
(31) Poutrel, Q.-A.; Blaker, J.; Soutis, C.; Tournilhac, F.; Gresil, M. Dicarboxylic acid-epoxy vitrimers: Influence of off-stoichiometric acid content on cure reactions and thermo-mechanical properties. Polym. Chem. 2020, 11, 5327-5338, DOI: $10.1039 /$ d0py00342e.

(32) Denissen, W.; Droesbeke, M.; Nicolaÿ, R.; Leibler, L.; Winne, J. M.; Du Prez, F. E.; Nicola, R.; Leibler, L.; Winne, J. M.; Du Prez, F. E. Chemical control of the viscoelastic properties of vinylogous urethane vitrimers. Nat. Commun. 2017, 8, 14857, DOI: $10.1038 /$ ncomms14857.

(33) Self, J. L.; Dolinski, N. D.; Zayas, M. S.; Read De Alaniz, J.; Bates, C. M. Brønstedacid-catalyzed exchange in polyester dynamic covalent networks. ACS Macro Lett. 2018, 7, 817-821, DOI: 10.1021 /acsmacrolett.8b00370.

(34) Demongeot, A.; Mougnier, S. J.; Okada, S.; Soulié-Ziakovic, C.; Tournilhac, F. Coordination and catalysis of $\mathrm{Zn}^{2+}$ in epoxy-based vitrimers. Polym. Chem. 2016, 7, 4486-4493, DOI: $10.1039 /$ c6py00752j.

(35) Fortman, D. J.; Brutman, J. P.; Cramer, C. J.; Hillmyer, M. A.; Dichtel, W. R. Mechanically activated, catalyst-free polyhydroxyurethane vitrimers. J. Am. Chem. Soc. 2015, 137, 14019-14022, DOI: $10.1021 /$ jacs.5b08084.

(36) Snyder, R. L.; Fortman, D. J.; De Hoe, G. X.; Hillmyer, M. A.; Dichtel, W. R. Reprocessable acid-degradable polycarbonate vitrimers. Macromolecules 2018, 51, 389-397, DOI: 10.1021 /acs.macromol. 7b02299.

(37) Lu, Y.-X. X.; Tournilhac, F.; Leibler, L.; Guan, Z. Making insoluble polymer networks malleable via olefin metathesis. J. Am. Chem. Soc. 2012, 134, 8424-8427, DOI: $10.1021 /$ ja303356z.

(38) Breuillac, A.; Kassalias, A.; Nicolaÿ, R. Polybutadiene vitrimers based on dioxaborolane 
chemistry and dual networks with static and dynamic cross-links. Macromolecules 2019 , 52, 7102-7113, DOI: 10.1021 /acs.macromol.9b01288.

(39) He, C.; Christensen, P. R.; Seguin, T. J.; Dailing, E. A.; Wood, B. M.; Walde, R. K.; Persson, K. A.; Russell, T. P.; Helms, B. A. Conformational entropy as a means to control the behavior of poly(diketoenamine) vitrimers in and out of equilibrium. Angew. Chemie - Int. Ed. 2020, 59, 735-739, DOI: 10.1002 /anie. 201912223.

(40) Zhou, Y.; Goossens, J. G.; Sijbesma, R. P.; Heuts, J. P. Poly(butylene terephthalate)/glycerol-based vitrimers via solid-state polymerization. Macromolecules 2017, 50, 6742-6751, DOI: 10.1021 /acs.macromol . 7b01142.

(41) Zhou, Y.; Goossens, J. G.; van den Bergen, S.; Sijbesma, R. P.; Heuts, J. P. In situ network formation in PBT vitrimers via processing-induced deprotection chemistry. Macromol. Rapid Commun. 2018, 39, 1800356, DOI: 10.1002 /marc. 201800356.

(42) Zhou, Y.; Groote, R.; Goossens, J. G.; Sijbesma, R. P.; Heuts, J. P. Tuning PBT vitrimer properties by controlling the dynamics of the adaptable network. Polym. Chem. 2019, 10, 136-144, DOI: $10.1039 /$ c8py01156g.

(43) Li, L.; Chen, X.; Jin, K.; Torkelson, J. M. Vitrimers designed both to strongly suppress creep and to recover original cross-link density after reprocessing: Quantitative theory and experiments. Macromolecules 2018, 51, 5537-5546, DOI: 10.1021 /acs.macromol.8b00922.

(44) Meng, F.; Saed, M. O.; Terentjev, E. M. Elasticity and relaxation in full and partial vitrimer networks. Macromolecules 2019, 52, 7423-7429, DOI: 10.1021 /acs.macromol.9b01123.

(45) Saed, M. O.; Gablier, A.; Terentjev, E. M. Liquid crystalline vitrimers with full or partial boronic-ester bond exchange. Adv. Funct. Mater. 2020, 30, 1906458, DOI: 10.1002 /adfm.201906458. 
(46) Self, J. L.; Sample, C. S.; Levi, A. E.; Li, K.; Xie, R.; de Alaniz, J. R.; Bates, C. M. Dynamic bottlebrush polymer networks: self-healing in super-soft materials. J. Am. Chem. Soc. 2020, 142, 7567-7573, DOI: $10.1021 /$ jacs.0c01467.

(47) Chen, X.; Li, L.; Wei, T.; Torkelson, J. M. Reprocessable polymer networks designed with hydroxyurethane dynamic cross-links: Effect of backbone structure on network morphology, phase segregation, and property recovery. Macromol. Chem. Phys. 2019, 220, 1900083, DOI: $10.1002 / \mathrm{macp} .201900083$.

(48) Lessard, J. J.; Scheutz, G. M.; Sung, S. H.; Lantz, K. A.; Epps, T. H.; Sumerlin, B. S. Block copolymer vitrimers. J. Am. Chem. Soc. 2020, 142, 283-289, DOI: $10.1021 /$ jacs.9b10360.

(49) Altuna, F. I.; Casado, U.; Dell'Erba, I. E.; Luna, L.; Hoppe, C. E.; Williams, R. J. J. Epoxy vitrimers incorporating physical crosslinks produced by self-association of alkyl chains. Polym. Chem. 2020, 11, 1337-1347, DOI: 10.1039 / C9PY01787A.

(50) Yang, Y.; Pei, Z.; Zhang, X.; Tao, L.; Wei, Y.; Ji, Y. Carbon nanotube-vitrimer composite for facile and efficient photo-welding of epoxy. Chem. Sci. 2014, 5, 3486-3492, DOI: $10.1039 / \mathrm{c} 4 \mathrm{sc} 00543 \mathrm{k}$.

(51) Legrand, A.; Soulié-Ziakovic, C. Silica-epoxy vitrimer nanocomposites. Macromolecules 2016, 49, 5893-5902, DOI: 10.1021 /acs.macromol . 6b00 0826.

(52) Chabert, E.; Vial, J.; Cauchois, J. P.; Mihaluta, M.; Tournilhac, F. Multiple welding of long fiber epoxy vitrimer composites. Soft Matter 2016, 12, 4838-4845, DOI: $10.1039 / \mathrm{c} 6 \mathrm{sm} 00257 \mathrm{a}$.

(53) Yan, P.; Zhao, W.; Jiang, L.; Wu, B.; Hu, K.; Yuan, Y.; Lei, J. Reconfiguration and shape memory triggered by heat and light of carbon nanotubepolyurethane vitrimer composites. $J$. Appl. Polym. Sci. 2018, 135, 45784, DOI: 10.1002 / app. 45784. 
(54) Spiesschaert, Y.; Taplan, C.; Stricker, L.; Guerre, M.; Winne, J. M.; Du Prez, F. E. Influence of the polymer matrix on the viscoelastic behaviour of vitrimers. Polym. Chem. 2020, 11, 5377-5385, DOI: $10.1039 /$ DOPY00114G.

(55) Snijkers, F.; Pasquino, R.; Maffezzoli, A. Curing and viscoelasticity of vitrimers. Soft Matter 2017, 13, 258-268, DOI: 10.1039 /C6SM0 0707 D.

(56) Wu, S.; Yang, H.; Huang, S.; Chen, Q. Relationship between reaction kinetics and chain dynamics of vitrimers based on dioxaborolane metathesis. Macromolecules 2020, 53, 11801190, DOI: 10.1021 /acs.macromol.9b02162.

(57) Hayashi, M.; Yano, R.; Takasu, A. Synthesis of amorphous low $T_{g}$ polyesters with multiple cooh side groups and their utilization for elastomeric vitrimers based on post-polymerization cross-linking. Polym. Chem. 2019, 10, 2047-2056, DOI: 10.1039 / C9PY 00293 F.

(58) Pritchard, R. H.; Redmann, A.-L. L.; Pei, Z.; Ji, Y.; Terentjev, E. M. Vitrification and plastic flow in transient elastomer networks. Polymer 2016, 95, 45-51, DOI: $10.1016 / j . p o l y m e r .2016 .04 .060$.

(59) Meng, F.; Pritchard, R. H.; Terentjev, E. M. Stress relaxation, dynamics, and plasticity of transient polymer networks. Macromolecules 2016, 49, 2843-2852, DOI: 10.1021 /acs.macromol.5b02667.

(60) Meng, F.; Terentjev, E. Transient network at large deformations: Elastic-plastic transition and necking instability. Polymers 2016, 8, 108, DOI: $10.3390 /$ polym8040108.

(61) Long, R.; Qi, H. J.; Dunn, M. L. Modeling the mechanics of covalently adaptable polymer networks with temperature-dependent bond exchange reactions. Soft Matter 2013, 9, 40834096, DOI: $10.1039 /$ c3sm27945f.

(62) Ma, J.; Mu, X.; Bowman, C. N.; Sun, Y.; Dunn, M. L.; Qi, H. J.; Fang, D. A photoviscoplas- 
tic model for photoactivated covalent adaptive networks. J. Mech. Phys. Solids 2014, 70, 84-103, DOI: $10.1016 / j$.jmps . 2014.05.008.

(63) Yu, K.; Shi, Q.; Wang, T.; Dunn, M. L.; Jerry Qi, H. A computational model for surface welding in covalent adaptable networks using finite-element analysis. J. Appl. Mech. 2016, 83,1-11, DOI: $10.1115 / 1.4033682$.

(64) Yu, K.; Shi, Q.; Li, H.; Jabour, J.; Yang, H.; Dunn, M. L.; Wang, T.; Qi, H. J. Interfacial welding of dynamic covalent network polymers. J. Mech. Phys. Solids 2016, 94, 1-17, DOI: $10.1016 / j \cdot j m p s .2016 .03 .009$.

(65) Sun, X.; Wu, H.; Long, R. Thermomechanics of a temperature sensitive covalent adaptable polymer with bond exchange reactions. Soft Matter 2016, 12, 8847-8860, DOI: $10.1039 / \mathrm{c} 6 \mathrm{sm} 01857 \mathrm{~b}$.

(66) Luo, C.; Shi, X.; Lei, Z.; Zhu, C.; Zhang, W.; Yu, K. Effects of bond exchange reactions and relaxation of polymer chains on the thermomechanical behaviors of covalent adaptable network polymers. Polymer 2018, 153, 43-51, DOI: $10.1016 / j . p o l y m e r .2018 .08 .001$.

(67) Jourdain, A.; Asbai, R.; Anaya, O.; Chehimi, M. M.; Drockenmuller, E.; Montarnal, D. Rheological properties of covalent adaptable networks with 1,2,3-triazolium cross-links: the missing link between vitrimers and dissociative networks. Macromolecules 2020, 53, 1884-1900, DOI: 10.1021 /acs.macromol .9b02204.

(68) Fang, H.; Fang, H.; Fang, H.; Ye, W.; Ding, Y.; Ding, Y.; Winter, H. H. Rheology of the critical transition state of an epoxy vitrimer. Macromolecules 2020, 53, 4855-4862, DOI: 10.1021 /acs.macromol.0c00843.

(69) Perego, A.; Khabaz, F. Volumetric and rheological properties of vitrimers: a hybrid molecular dynamics and Monte Carlo simulation study. Macromolecules 2020, 53, 8406-8416, DOI: 10.1021 /acs.macromol .0c01423. 
(70) Smallenburg, F.; Leibler, L.; Sciortino, F. Patchy particle model for vitrimers. Phys. Rev. Lett. 2013, 111, 188002, DOI: $10.1103 /$ PhysRevLett.111.188002.

(71) Rovigatti, L.; Nava, G.; Bellini, T.; Sciortino, F. Self-dynamics and collective swap-driven dynamics in a particle model for vitrimers. Macromolecules 2018, 51, 1232-1241, DOI: 10.1021 /acs.macromol.7b02186.

(72) Ciarella, S.; Sciortino, F.; Ellenbroek, W. G. Dynamics of vitrimers: Defects as a highway to stress relaxation. Phys. Rev. Lett. 2018, 121, 058003, DOI: $10.1103 /$ PhysRevLett.121.058003.

(73) Shivokhin, M. E.; Narita, T.; Talini, L.; Habicht, A.; Seiffert, S.; Indei, T.; Schieber, J. D. Interplay of entanglement and association effects on the dynamics of semidilute solutions of multisticker polymer chains. J. Rheol. 2017, 61, 1231-1241, DOI: $10.1122 / 1.4997740$.

(74) Green, M. S.; Tobolsky, A. V. A new approach to the theory of relaxing polymeric media. J. Chem. Phys. 1946, 14, 80-92, DOI: $10.1063 / 1.1724109$.

(75) Yamamoto, M. The visco-elastic properties of network structure I. General formalism. J. Phys. Soc. Jpn. 1956, 11, 413-421, DOI: 10 .1143/ JP S J.11. 413.

(76) Lodge, A. A network theory of flow birefringence and stress in concentrated polymer solutions. Trans. Faraday Soc. 1956, 52, 120-130.

(77) Tanaka, F.; Edwards, S. F. Viscoelastic properties of physically crosslinked networks. 1. Transient network theory. Macromolecules 1992, 25, 1516-1523, DOI: $10.1021 / \mathrm{ma} 00031 \mathrm{a} 024$.

(78) Wientjes, R. H. W.; Jongschaap, R. J. J.; Duits, M. H. G.; Mellema, J. A new transient network model for associative polymer networks. J. Rheol. 1999, 43, 375-391, DOI: $10.1122 / 1.551039$. 
(79) Indei, T.; Takimoto, J.-i. Linear viscoelastic properties of transient networks formed by associating polymers with multiple stickers. J. Chem. Phys. 2010, 133, 194902, DOI: $10.1063 / 1.3498779$.

(80) Baxandall, L. G. Dynamics of reversibly crosslinked chains. Macromolecules 1989, 22, 1982-1988, DOI: $10.1021 / \mathrm{ma} 00194 \mathrm{a} 076$.

(81) Rouse, P. R. A theory of the linear viscoelastic properties of dilute solutions of coiling polymers. J. Chem. Phys. 1953, 21, 1272-1280.

(82) Leibler, L.; Rubinstein, M.; Colby, R. H. Dynamics of reversible networks. Macromolecules 1991, 24, 4701-4707, DOI: 10.1021 /ma $00016 a 034$.

(83) Leibler, L.; Rubinstein, M.; Colby, R. H. Dynamics of telechelic ionomers. Can polymers diffuse large distances without relaxing stress? J. Phys. II 1993, 3, 1581-1590, DOI: $10.1051 /$ jp2:1993219.

(84) Rubinstein, M.; Semenov, A. N. Thermoreversible gelation in solutions of associating polymers. 2. Linear dynamics. Macromolecules 1998, 31, 1386-1397, DOI: $10.1021 / \mathrm{ma} 970617+$.

(85) Rubinstein, M.; Semenov, A. N. Dynamics of entangled solutions of associating polymers. Macromolecules 2001, 34, 1058-1068, DOI: 10.1021 /ma 0013049.

(86) Chen, Q.; Tudryn, G. J.; Colby, R. H. Ionomer dynamics and the sticky Rouse model. J. Rheol. 2013, 57, 1441-1462, DOI: $10.1122 / 1$. 4818868.

(87) Zhang, Z.; Chen, Q.; Colby, R. H. Dynamics of associative polymers. Soft Matter 2018, 14, 2961-2977, DOI: $10.1039 /$ C8SM0 0044 A.

(88) Tripathi, A.; Tam, K. C.; McKinley, G. H. Rheology and dynamics of associative polymers in shear and extension: Theory and experiments. Macromolecules 2006, 39, 1981-1999, DOI: $10.1021 / \mathrm{ma} 051614 \mathrm{x}$. 
(89) Ahmadi, M.; Hawke, L. G. D.; Goldansaz, H.; van Ruymbeke, E. Dynamics of entangled linear supramolecular chains with sticky side groups: Influence of hindered fluctuations. Macromolecules 2015, 48, 7300-7310, DOI: 10 . 1021 / acs . macromol . 5b 00733.

(90) Hawke, L. G. D.; Ahmadi, M.; Goldansaz, H.; van Ruymbeke, E. Viscoelastic properties of linear associating poly(n-butyl acrylate) chains. J. Rheol. 2016, 60, 297-310, DOI: $10.1122 / 1.4942231$.

(91) Spruijt, E.; Cohen Stuart, M. A.; Van Der Gucht, J. Linear viscoelasticity of polyelectrolyte complex coacervates. Macromolecules 2013, 46, 1633-1641, DOI: $10.1021 / \mathrm{ma} 301730 \mathrm{n}$.

(92) Syed, V. M.; Srivastava, S. Time-ionic strength superposition: A unified description of chain relaxation dynamics in polyelectrolyte complexes. ACS Macro Letters 2020, 9, 1067-1073, DOI: 10.1021 /acsmacrolett.0c00252.

(93) Morin, F. J.; Puppo, M. L.; Laaser, J. Decoupling salt- and polymer-dependent dynamics in polyelectrolyte complex coacervates via salt addition. 2020; https://chemrxiv.org/articles/preprint/Decoupling_salt-_ and_Polymer-Dependent_Dynamics_in_Polyelectrolyte_Complex_ Coacervates_via_Salt_Addition/12469556/1.

(94) Tang, S.; Wang, M.; Olsen, B. D. Anomalous self-diffusion and sticky Rouse dynamics in associative protein hydrogels. J. Am. Chem. Soc. 2015, 137, 3946-3957, DOI: $10.1021 /$ jacs.5b00722.

(95) Colby, R. H.; Zheng, X.; Rafailovich, M. H.; Sokolov, J.; Peiffer, D. G.; Schwarz, S. A.; Strzhemechny, Y.; Nguyen, D. Dynamics of lightly sulfonated polystyrene ionomers. Phys. Rev. Lett. 1998, 81, 3876-3879, DOI: 10.1103 / Phys RevLett . 81 . 3876.

(96) de Gennes, P. G. Kinetics of diffusioncontrolled processes in dense polymer systems. I. Nonentangled regimes. J. Chem. Phys. 1982, 76, 3316-3321, DOI: $10.1063 / 1$. 443328. 
(97) Bird, R.; Armstrong, R.; Hassager, O. Dynamics of polymeric liquids, Vol. 1, Fluid mechanics; John Wiley\& Sons: New York, 1987.

(98) Larson, R. G. Constitutive equations for polymer melts and solutions; ButterworthHeinemann, 1988; DOI: https: / /doi .org/10.1016/C2013-0-04284-3.

(99) Doi, M.; Edwards, S. F. The theory of polymer dynamics; Clarendon Press: Oxford, 1986.

(100) Provencher, S. W. An eigenfunction expansion method for the analysis of exponential decay curves. J. Chem. Phys. 1976, 64, 2772-2777, DOI: $10.1063 / 1$. 432601.

(101) Baumgaertel, M.; Winter, H. H. Determination of discrete relaxation and retardation time spectra from dynamic mechanical data. Rheol. Acta 1989, 28, 511-519, DOI: $10.1007 / \mathrm{BF} 01332922$.

(102) Takeh, A.; Shanbhag, S. A computer program to extract the continuous and discrete relaxation spectra from dynamic viscoelastic measurements. Appl. Rheol. 2013, 23, 24628.

(103) Shanbhag, S. pyReSpect: A computer program to extract discrete and continuous spectra from stress relaxation experiments. Macromol. Theory Simul. 2019, 1900005, DOI: 10.1002 /mats. 201900005 .

(104) Shanbhag, S. Relaxation spectra using nonlinear Tikhonov regularization with a Bayesian criterion. Rheol. Acta 2020, 59, 509-520, DOI: 10 . 1007 / s $00397-020$-01212-w.

(105) Hansen, D. R.; Shen, M. Viscoelastic retardation time computations for homogeneous block copolymers. Macromolecules 1975, 8, 343-348, DOI: 10.1021 /ma 60045 a 020.

(106) Stockmayer, W. H.; Kennedy, J. W. Viscoelastic spectrum of free-draining block copolymers. Macromolecules 1975, 8, 351-355, DOI: 10 . 1021 /ma 60045 a 022.

(107) Wang, F. W.; DiMarzio, E. A. The dynamics of block-copolymer molecules in solution. The free-draining limit. Macromolecules 1975, 8, 356-360, DOI: 10 . 1021 /ma 60045 a 023. 
(108) Rubinstein, M.; Helfand, E.; Pearson, D. S. Theory of polydispersity effects of polymer rheology: Binary distribution of molecular weights. Macromolecules 1987, 20, 822-829, DOI: $10.1021 / \mathrm{ma} 00170 \mathrm{a} 021$.

(109) Hung, J.-H.; Mangalara, J. H.; Simmons, D. S. Heterogeneous Rouse model predicts polymer chain translational normal mode decoupling. Macromolecules 2018, 51, 2887-2898, DOI: 10.1021 /acs.macromol.8b00135.

(110) Jiang, N.; Zhang, H.; Tang, P.; Yang, Y. Linear viscoelasticity of associative polymers: Sticky Rouse model and the role of bridges. Macromolecules 2020, 53, 3438-3451, DOI: $10.1021 /$ acs.macromol.0c00312.

(111) Dean, P. Vibrations of glass-like disordered chains. Proc. Phys. Soc. 1964, 84, 727-744, DOI: $10.1088 / 0370-1328 / 84 / 5 / 310$.

(112) Chen, Q.; Liang, S.; Shiau, H.-s.; Colby, R. H. Linear viscoelastic and dielectric properties of phosphonium siloxane ionomers. ACS Macro Lett. 2013, 2, 970-974, DOI: $10.1021 / \mathrm{mz} 400476 \mathrm{w}$.

(113) Laidler, K. J. The development of the Arrhenius equation. J. Chem. Educ. 1984, 61, 494, DOI: $10.1021 /$ ed061p494.

(114) Criado, J.; Pérez-Maqueda, L.; Sánchez-Jiménez, P. Dependence of the preexponential factor on temperature. J. Therm. Anal. Calorim. 2005, 82, 671-675.

(115) Oeser, R.; Ewen, B.; Richter, D.; Farago, B. Dynamic fluctuations of crosslinks in a rubber: a neutron-spin-echo study. Phys. Rev. Lett. 1988, 60, 1041-1044, DOI: 10.1103/PhysRevLett.60.1041.

(116) Williams, M. L.; Landel, R. F.; Ferry, J. D. The temperature dependence of relaxation mechanisms in amorphous polymers and other glass-forming liquids. J. Am. Chem. Soc. 1955, 77, 3701-3707, DOI: $10.1021 /$ ja01619a008. 
(117) Ferry, J. Viscoelastic properties of polymers, $3^{\text {rd }}$ ed.; John Wiley \& Sons Inc: Hoboken, NY, 1980.

(118) Fuchs, K.; Friedrich, C.; Weese, J. Viscoelastic properties of narrow-distribution poly(methyl methacrylates). Macromolecules 1996, 29, 5893-5901, DOI: $10.1021 / \mathrm{ma} 951385 \mathrm{~m}$.

(119) Zhang, Z.; Huang, C.; Weiss, R. A.; Chen, Q. Association energy in strongly associative polymers. J. Rheol. 2017, 61, 1199-1207, DOI: 10.1122/1.4997586.

(120) Camino, G.; Lomakin, S.; Lazzari, M. Polydimethylsiloxane thermal degradation Part 1. Kinetic aspects. Polymer 2001, 42, 2395 - 2402, DOI: https://doi.org/10.1016/s0032-3861(00)00652-2.

(121) Chrissafis, K.; Bikiaris, D. Can nanoparticles really enhance thermal stability of polymers? Part I: An overview on thermal decomposition of addition polymers. Thermochim. Acta 2011,523,1-24, DOI: https://doi.org/10.1016/j.tca.2011.06.010.

(122) Brassinne, J.; Cadix, A.; Wilson, J.; van Ruymbeke, E. Dissociating sticker dynamics from chain relaxation in supramolecular polymer networks - The importance of free partner! $J$. Rheol. 2017, 61, 1123-1134, DOI: 10.1122/1.4997594.

(123) Gold, B. J.; Hvelmann, C. H.; Lhmann, N.; Szkely, N. K.; PyckhoutHintzen, W.; Wischnewski, A.; Richter, D. Importance of compact random walks for the rheology of transient networks. ACS Macro Lett. 2017, 6, 73-77, DOI: 10.1021/acsmacrolett.6b00880.

(124) Stukalin, E. B.; Cai, L.-H.; Kumar, N. A.; Leibler, L.; Rubinstein, M. Self-healing of unentangled polymer networks with reversible bonds. Macromolecules 2013, 46, 7525-7541, DOI: $10.1021 / \mathrm{ma} 401111 \mathrm{n}$. 
(125) Schapery, R.; Park, S. Methods of interconversion between linear viscoelastic material functions. Part II - an approximate analytical method. Int. J. Solids Struct. 1999, 36, 1677 - 1699, DOI: https://doi.org/10.1016/s0020-7683(98)00060-2.

(126) Loy, R. J.; de Hoog, F. R.; Anderssen, R. S. Interconversion of Prony series for relaxation and creep. J. Rheol. 2015, 59, 1261-1270, DOI: $10.1122 / 1$. 4929398.

(127) Flory, P. J.; Rehner, J. Statistical mechanics of crossLinked polymer networks I. Rubberlike elasticity. J. Chem. Phys. 1943, 11, 512-520, DOI: $10.1063 / 1.1723791$.

(128) Flory, P. J. Molecular size distribution in three dimensional polymers. I. Gelation. J. Am. Chem. Soc. 1941, 63, 3083-3090, DOI: 10.1021 / ja01856a061.

(129) Stockmayer, W. H. Theory of molecular size distribution and gel formation in branched polymers II. General cross linking. J. Chem. Phys. 1944, 12, 125-131, DOI: $10.1063 / 1.1723922$.

(130) Zhou, H.; Woo, J.; Cok, A. M.; Wang, M.; Olsen, B. D.; Johnson, J. A. Counting primary loops in polymer gels. Proc. Natl. Acad. Sci. 2012, 109, 19119-19124, DOI: 10.1073 /pnas.1213169109.

(131) Schieber, J. D.; Andreev, M. Entangled polymer dynamics in equilibrium and flow modeled through slip links. Ann. Rev. Chem. Biomol. Engg. 2014, 5, 367-381, DOI: $10.1146 /$ annurev-chembioeng-060713-040252.

(132) Masubuchi, Y. Simulating the flow of entangled polymers. Ann. Rev. Chem. Biomol. Engg. 2014, 5, 11-33, DOI: 10.1146 /annurev-chembioeng-060713-040401, PMID: 24498953.

(133) Shanbhag, S. Fast slip link model for bidisperse linear polymer melts. Macromolecules 2019, 52, 3092-3103, DOI: 10.1021 /acs.macromol . 8b02367. 
(134) Müller, M.; Dardin, A.; Seidel, U.; Balsamo, V.; Iván, B.; Spiess, H. W.; Stadler, R. Junction dynamics in telechelic hydrogen bonded polyisobutylene networks. Macromolecules 1996, 29, 2577-2583, DOI: 10.1021 /ma950984q.

(135) Adzima, B. J.; Aguirre, H. A.; Kloxin, C. J.; Scott, T. F.; Bowman, C. N. Rheological and chemical analysis of reverse gelation in a covalently cross-linked Diels-Alder polymer network. Macromolecules 2008, 41, 9112-9117, DOI: 10.1021 /ma801863d, PMID: 20711364.

(136) Stadler, F. J.; Pyckhout-Hintzen, W.; Schumers, J.-M.; Fustin, C.-A.; Gohy, J.-F.; Bailly, C. Linear viscoelastic rheology of moderately entangled telechelic polybutadiene temporary networks. Macromolecules 2009, 42, 6181-6192, DOI: 10 . 1021/ma 802488 a.

(137) Weiss, R. A.; Zhao, H. Rheological behavior of oligomeric ionomers. J. Rheol. 2009, 53, 191-213, DOI: $10.1122 / 1.3003570$. 


\section{Graphical TOC Entry}

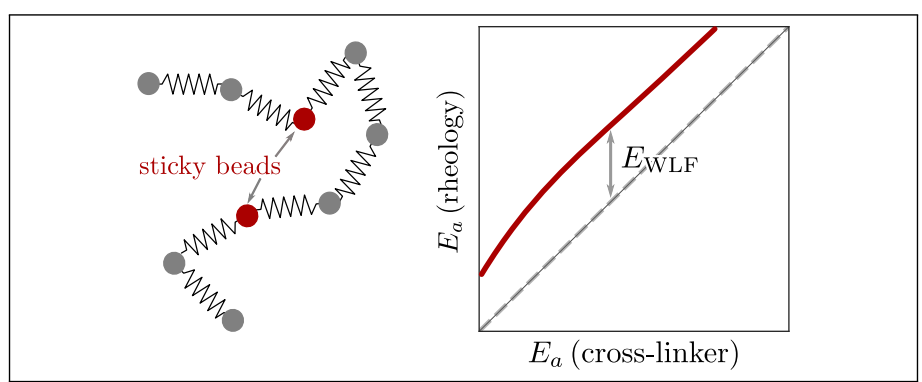




\title{
Supporting Information
}

\section{Unentangled Vitrimer Melts: Interplay between Chain Relaxation and Cross-link Exchange Controls Linear Rheology}$$
\text { Ralm Ricarte }{ }^{*, \dagger} \text { and Sachin Shanbhag }{ }^{*, \ddagger}
$$ \\ ${ }^{1}$ Department of Chemical and Biomedical Engineering, FAMU-FSU College of Engineering, Tallahassee, \\ FL 32310, USA. \\ ${ }^{2}$ Department of Scientific Computing, Florida State University, Tallahassee, FL 32306, USA.
}

\section{Contents}

1 Mathematical Notation for Different Timescales

2 Experimental Data on Vitrimer Systems

${ }^{\dagger}$ rricarte@eng.famu.fsu.edu

${ }^{\ddagger}$ sshanbhag@fsu.edu 


\section{Mathematical Notation for Different Timescales}

All timescales are denoted by the Greek letter " $\tau$ "; superscripts, subscripts, and accents are used to distinguish them. The table below summarizes the different timescales used in the paper.

\begin{tabular}{cll}
\hline symbol & definition & comment \\
\hline$\tau_{b}$ & (drag of bead)/(spring constant) & $\zeta / k$ \\
$\tau$ & elementary Rouse time & $\tau_{b} / 8$ \\
$\tau_{i}$ & Rouse spectrum $1 \leq i \leq N-1$ & eqn (4) \\
$\tau_{1}$ & longest Rouse time & $\tau_{i}$ with $i=1$ \\
$\hat{\tau}$ & approx. elementary Rouse time assuming $N \gg 1$ & $\tau_{b} /\left(2 \pi^{2}\right)$ \\
$\hat{\tau}_{i}$ & approx. Rouse spectrum assuming $N \gg 1$ & eqn $(5)$ \\
$\tau_{\eta}$ & average relaxation time & $\left(\sum_{i} \tau_{i}\right) / N$ \\
$\tau_{\eta}^{R}$ & $\tau_{\eta}$ for standard Rouse model & eqn $(8)$ \\
$\tau_{x}$ & lifetime of XL & eqn $(14)$ \\
$\tau_{x}^{0}$ & Arrhenius prefactor for $\tau_{x}$ & eqn $(14) ;$ we assume $\tau_{x}^{0}=2 \tau$ \\
$\tau^{*}$ & characteristic relaxation time & $\tau_{\eta}$ or $\tau_{1}$, depending on use case \\
\hline
\end{tabular}

Table S1: Symbols used for different timescales. 


\section{Experimental Data on Vitrimer Systems}

The following table compiles vitrimer activation energy data from sources in which $E_{a}^{\mathrm{rh}}$ and $E_{a}^{\mathrm{sm}}$ were measured separately. In all cases, the vitrimer chains are nominally entangled. Furthermore, in nearly all of these studies the characteristic relaxation time $\tau^{*}$ is obtained from stress relaxation, using the $1 / e$ criterion, viz. $G\left(\tau^{*}\right) / G(0)=1 / e$, to determine $E_{a}^{\mathrm{rh}}$. This likely leads to underestimation of the true value. In fact, reference 9 even directly shows that this criterion leads to lower $E_{a}^{\text {rh }}$ compared to values determined from viscosity and $\tau_{1}$. In the case of the reference 56 , the model system contains small molecule dioxaborolanes that participate in exchange reactions, which violates the assumptions made in the IHR theory.

\begin{tabular}{lllccl}
\hline Ref & Backbone & Cross-linker & $\begin{array}{c}E_{a}^{\mathrm{sm}} \\
\mathrm{kJ} / \mathrm{mol}\end{array}$ & $\begin{array}{c}E_{a}^{\mathrm{rh}} \\
\mathrm{kJ} / \mathrm{mol}\end{array}$ & $E_{a}^{\mathrm{rh}}$ determination \\
\hline 9 & PMMA & Dioxaborolane & 15 & 43.2 & $\tau^{*}$ vs. $1 / T .^{b}$ \\
9 & PMMA & Dioxaborolane & 15 & 62.3 & $\eta_{0}$ vs. $1 / T$, measured by stress relaxation \\
9 & PMMA & Dioxaborolane & 15 & 76.7 & $\tau_{1}$ vs. $1 / T$, measured by creep \\
10 & PS & Silyl ether & 35 & 81 & $\tau^{*}$ vs. $1 / T .^{b}$ \\
10 & PS & Silyl ether & 65 & 174 & $\tau^{*}$ vs. $1 / T .^{b}$ \\
15 & PMMA & Vinylogous urethane & 80 & 102 & $\tau^{*}$ vs. $1 / T .^{b}$ \\
16 & PS & Vinylogous urethane & 80 & 165 & $\tau^{*}$ vs. $1 / T .^{b}$ \\
56 & PHMA $^{a}$ & Dioxaborolane & 15 & 52 & Time-temperature superposition shift factors \\
& & & & & vs. $1 / T$, measured by SAOS \\
\hline
\end{tabular}

${ }^{a}$ poly(hexyl methacrylate)

${ }^{b} \tau^{*}$ is obtained from stress relaxation, using the $1 / e$ criterion, viz. $G\left(\tau^{*}\right) / G(0)=1 / e$.

Table S2: Characterization of experimental datasets 